\title{
A Stable Maximum-Entropy Meshless Method for Analysis of Porous Media
}

\author{
M. Nazem*, M. Kardani ${ }^{*}$, B. Bienen ${ }^{+}$, M. Cassidy ${ }^{+}$ \\ *Australian Research Council Centre of Excellence for Geotechnical Science and Engineering, University of \\ Newcastle, NSW, Australia \\ ${ }^{+}$Centre for Offshore Foundation Systems and Australian Research Council Centre of Excellence for Geotechnical \\ Science and Engineering, University of Western Australia, WA, Australia
}

\begin{abstract}
Consolidation analysis of saturated porous media demands the coupling of solid displacements with the pore fluid pressure via the equilibrium and the continuity of mass. In this paper, a stable numerical procedure is presented for coupled analysis of consolidation problems in geotechnical engineering. The numerical framework is based on the Element-Free Galerkin method and the principle of Maximum Entropy. Identical shape functions are employed for approximating the displacement field as well as the pore fluid pressure field. The proposed method is used for analysing several consolidation problems assuming elastic and elastoplastic soil behaviour. The numerical results indicate that the proposed Maximum-Entropy Meshless method based on the maximum entropy shape functions is able to provide stable and robust solutions for consolidation problems in porous media.
\end{abstract}

Keywords: Consolidation, Meshless, Maximum entropy, Numerical Analysis

\section{INTRODUCTION}

Meshless methods have significantly progressed during the past few decades. In these methods a set of nodes is used to discretise the domain of a problem and, hence, unlike the finite element (FE) method, connectivity between elements and nodal points does not exist. This concept implies that meshless methods are more appropriate than the FE method for tackling geotechnical problems including extremely large deformations, discontinuities due to crack propagation and separation of materials, moving boundary conditions, and strain localisation. It is noteworthy that in geotechnical applications, the continuum usually includes a solid phase (soil particles) and a fluid phase (water), demanding a robust and stable computational method to model two-phase response. Among others, the Element-Free Galerkin 
(EFG) method, developed by Belytschko et al. [1], has attracted significant attention for solving geotechnical problems, due to its robustness, relatively high accuracy, and superior convergence, e.g. see [14][22][31][33]. In the original EFG method, Moving Least Square (MLS) shape functions were employed to approximate unknown field variables [1]. MLS shape functions do not satisfy the Kronecker delta property, creating technical difficulties and extra computational challenge for imposing the essential boundary conditions. Recently, the concept of maximum entropy (max-ent) shape functions was introduced to the EFG method, which facilitates application of boundary conditions [1][23][26]. This technique is usually referred to as the Maximum-Entropy Meshless (MEM) method. Due to their robustness, the max-ent shape functions within the framework of the EFG method have been widely used in engineering problems. Examples include incompressible elasticity [15], nonlinear analysis of reinforced concrete structures [18], and analysis of thin shells [9]. More recently, these shape functions have been employed for adaptive meshless analysis [30] as well as combined FE-MEM methods of analysis of nonlinear solid problems [31].

Consolidation analysis of porous media by meshless methods has attracted significant attention. Among others, the consolidation phenomenon has been investigated using the EFG method [10][13][14][19][22], and the Radial Point Interior (RPI) method [7][6][34][35]. In such analyses the unknown variables include displacements, $\mathbf{u}$, as well as the pore fluid pressures, $\mathbf{p}$. The accurate prediction of pore water pressure has faced difficulties, for which stabilisation techniques have been proposed. The instabilities in a coupled u-p analysis are mainly due to (a) the time integration scheme, (b) the inconsistency between the order of shape functions used for interpolating displacements and those employed for approximating pore water pressures. Within the framework of the RPI method, Khoshghalb and Khalili [7] developed a three-point time discretisation technique which is second-order accurate and avoids the spurious ripple effects observed in a two-point integration scheme. Shibata and Mukarami [22] observed instability in consolidation analysis by the EFG method and introduced a stabilising term into the weak form of governing equations. The main advantage of this technique is that the interpolation fields in the pore pressure field do not reduce the accuracy of numerical results. Nonetheless, the method demands extra computational challenge [22]. Regardless of the numerical method, another source of instability in analysis of consolidation problems is often due to the inconsistency between the degrees of displacement field and the pore water pressure field. Oliaei et al. [14] demonstrated that the same order of MLS shape functions for displacement and pore water pressure provides stability as well as accuracy of numerical results provided an implicit time-integration scheme is employed.

In this study, the MEM method is further extended to allow fully coupled analysis of fully saturated porous media. It is demonstrated that the proposed method is intrinsically stable (no stabilisation technique is employed), does not require special treatment of the essential boundary conditions, and can successfully tackle elasto-plastic consolidation problems in fully saturated porous media. This is mainly 
due to the fact that the max-ent shape functions tend to be much less sensitive to the discretisation of the domain as well as the diversity in values of the interpolation field [15].

The outline of the paper is as follows. The equations governing the consolidation of a porous continuum are presented within the framework of the MEM method in Section 2. Then, an implicit scheme based on the Backward Euler method for integrating the global equations is briefly discussed in Section 3. The maximum entropy principle and the corresponding shape functions are introduced in Section 4. In Section 5 , several numerical examples are presented to validate the formulation and to demonstrate the stability of the proposed method. The key outcomes of this study and the conclusions drawn are summarised in Section 6.

\section{GOVERNING EQUATIONS}

In this section the equations governing the nonlinear behaviour of a two-phase porous medium, assuming small deformations, are presented. In geotechnical problems, deformations are usually coupled with the dissipation of excess pore fluid pressure. Numerical analysis of such problems requires the coupling of the equilibrium with the continuity equation through the principle of effective stresses and Darcy's law. Consider the continuum shown in Figure 1, where the problem domain $\Omega$ is bounded by domain boundary $\Gamma$. The equilibrium is satisfied provided that

$$
\sigma_{i j, j}+b_{i}=0
$$

where $\sigma$ denotes the total Cauchy stress vector, $b$ is the body force vector, and a comma in the subscript represents a partial derivative with respect to the indicated variable. Denoting $v_{\mathrm{s}}$ as the velocity of soil particles and $\tilde{v}$ as the superficial velocity of the fluid relative to the soil skeleton, and assuming that the soil solids and the pore water are much less compressible than the soil skeleton, the conservation of mass can be expressed by

$$
\frac{\partial v_{\mathrm{si}}}{\partial x_{i}}+\frac{\partial \tilde{v}_{i}}{\partial x_{i}}=0
$$

The boundary conditions in Equations (1) and (2) are

$$
\begin{array}{ll}
\sigma_{i j} \cdot \bar{n}_{j}=T_{i} & \forall x \in \Gamma_{T} \\
u_{i}=\bar{u}_{i} & \forall x \in \Gamma_{u} \\
p_{i}=\bar{p}_{i} & \forall x \in \Gamma_{p} \\
q=n_{s} \tilde{v} \cdot_{i} \bar{n}_{i} & \forall x \in \Gamma_{q}
\end{array}
$$

where $\bar{n}$ is the vector of unit outward normal at a point $x$ on $\Gamma, T$ represents the prescribed traction on the traction boundary $\Gamma_{T}, u$ is the displacement vector, $\bar{u}$ denotes the prescribed displacement on the displacement boundary $\Gamma_{u}, p$ is the pore water pressure vector, $\bar{p}$ represents the prescribed pore water 
pressure on the pore water pressure boundary $\Gamma_{p}, q$ is the prescribed flow on boundary $\Gamma_{q}$, and $n_{s}$ is the porosity of soil.

In the original EFG method, the Moving Least Square (MLS) shape functions were employed to approximate unknown field variables [1]. The MLS shape functions do not satisfy the Kronecker delta property, creating technical difficulties and extra computational challenge for imposing the essential boundary conditions. Maximum entropy (max-ent) shape functions [23] provide an alternative way to interpolate the unknown variables while satisfying the Kronecker delta property. Therefore, the weak form of Equation (1) can be written as

$$
\int_{\Omega} \sigma_{i j} \delta u_{i, j} d \Omega+\int_{\Omega} b_{i} \delta u_{i} d \Omega+\int_{\Gamma_{T}} T_{i} \delta u_{i} d \Gamma_{T}=0
$$

where $\delta u_{i}$ denotes the vector of virtual displacements. The principle of effective stress relates the total Cauchy stress to the effective Cauchy stress, $\sigma^{\prime}$, and the pore water pressure, $p$,

$$
\sigma_{i j}=\sigma_{i j}^{\prime}+\delta_{i j} p_{i j}
$$

where $\delta_{i j}$ is the Kronecker-delta. Given an incremental strain in the solid phase, $d \varepsilon_{i j}$, and assuming small deformations, the effective stress is obtained by solving

$$
d \sigma_{i j}^{\prime}=D_{i j k l}^{\prime e p} d \varepsilon_{k l}=D_{i j k l}^{\prime e p} d u_{k, l}
$$

in which $D^{\prime e p}$ is the material constitutive matrix, and depends on the drained properties of the soil skeleton. The elastoplastic constitutive matrix $D^{\prime e p}$ is defined by (see e.g. [24][12])

$$
D_{i j k l}^{\prime e p}=D_{i j k l}^{\prime e}-\frac{D_{i j m n}^{\prime e} \cdot \frac{\partial f}{\partial \sigma_{m n}^{\prime}} \cdot D_{k l p q}^{\prime e} \cdot \frac{\partial g}{\partial \sigma_{p q}^{\prime}}}{\frac{\partial f}{\partial \sigma_{p q}^{\prime}} \cdot D_{p q r s}^{\prime e} \cdot \frac{\partial f}{\partial \sigma_{r s}^{\prime}}-\frac{\partial f}{\partial \kappa_{m}} \cdot B_{m}(\sigma, \kappa)}
$$

where $D^{\prime e}$ is the elastic stress-strain matrix, $f$ represents the yield surface, $g$ is the plastic potential surface, $\kappa$ denotes a set of hardening parameters and $B$ is a function derived from the hardening laws. In conventional elastoplastic constitutive laws, the elastic constitutive matrix is independent of the stresses and is obtained by 


$$
\mathbf{D}^{\prime e}=\frac{E^{\prime}\left(1-v^{\prime}\right)}{\left(1+v^{\prime}\right)\left(1-2 v^{\prime}\right)}\left[\begin{array}{cccccc}
1 & \frac{v^{\prime}}{1-v^{\prime}} & \frac{v^{\prime}}{1-v^{\prime}} & 0 & 0 & 0 \\
1 & \frac{v^{\prime}}{1-v^{\prime}} & 0 & 0 & 0 \\
& 1 & 0 & 0 & 0 \\
\text { Sym } & \frac{1-2 v^{\prime}}{2\left(1-v^{\prime}\right)} & 0 & 0 \\
& & \frac{1-2 v^{\prime}}{2\left(1-v^{\prime}\right)} & 0 \\
& & \frac{1-2 v^{\prime}}{2\left(1-v^{\prime}\right)}
\end{array}\right]
$$

where $E^{\prime}$ is the drained Young's modulus and $v^{\prime}$ represents the drained Poisson's ratio. It is notable that if the soil behaviour is elastic the second term on right hand side of Equation (7) will be zero.

Equation (4) is a weak form defined over the entire problem domain $\Omega$. The unknown field values in Equation (4) include the displacements of the solid phase as well as the pore water pressure. In the finite element method, if possible, different shape functions are used for interpolating the displacements and the pore water pressure, since pore water pressures are proportional to the gradient of displacements [16]. For example, if 6-noded triangular elements with quadratic shape functions are used for approximating the displacement field, the values of pore water pressure will be interpolated by using the shape functions of a linear triangular element. However, this consistency between the displacements and stresses (pore pressures) cannot be simply satisfied for higher order elements, leading the analyst to employ identical interpolation functions for both unknown nodal fields. A similar challenge is encountered when using the meshless methods. In this study, we employ the max-ent functions for interpolating the displacements as well as the pore water pressures at field nodes. In order to conduct the integration in Equation (4) the problem domain is firstly represented by a set of field nodes, as shown in Figure 1. Then, a background mesh containing the integration points is used to evaluate the integrals. Assuming $\Phi_{i}$ represents the matrix of max-ent shape function of a field node $i$ (see Section 4 for max-ent functions), the approximated displacement values of a point of interest $x, u^{h}$, and the approximated pore water pressures, $p^{h}$, can be obtained by

$$
\begin{aligned}
& u^{h}(x)=\sum_{i=1}^{n} \Phi_{i} u_{i} \\
& p^{h}(x)=\sum_{i=1}^{n} \Phi_{i} p_{i}
\end{aligned}
$$

where $n$ is the number of field nodes at the support domain of point $x$, and $u_{i}$ and $p_{i}$ represent, respectively, the nodal values of displacements and the pore water pressures. Using Equations (4)-(9), the rate form of the nonlinear system of equations that satisfies equilibrium can be expressed by 


$$
\dot{\mathbf{F}}^{i n t}=\mathbf{K}^{e p} \dot{\mathbf{u}}+\mathbf{L} \dot{\mathbf{p}}=\dot{\mathbf{F}}^{e x t}
$$

where $\mathbf{K}^{e p}$ and $\mathbf{L}$ are the stiffness and coupling matrices respectively, $\mathbf{F}^{\text {ext }}$ is the external force vector, $\mathbf{F}^{\text {int }}$ represents the internal force vector and a superimposed dot denotes the time derivative of a variable. Detailed expressions for $\mathbf{K}^{e p}, \mathbf{L}$ and $\mathbf{F}^{\text {int }}$ are provided in the Appendix.

Darcy's Law states that the superficial velocity of the fluid relative to the soil skeleton is proportional to the hydraulic gradient

$$
\tilde{v}_{i}=-\gamma_{w}^{-1} k_{i j}\left(\frac{\partial p}{\partial x_{j}}-b_{\mathrm{wj}}\right)
$$

where $k$ represents the permeability tensor, $\gamma_{w}$ is the unit weight of water, and, assuming that gravity acts in the negative 2-coordinate $(y)$ direction, $b_{w}=\left\{\begin{array}{llll}0 & \gamma_{w} & 0\end{array}\right\}^{T}$. Combining Equation (2) with Equation (11), the rate form of the continuity equation is obtained according to

$$
\mathbf{L}^{\mathrm{T}} \dot{\mathbf{u}}+\dot{\mathbf{H}} \mathbf{p}=\dot{\mathbf{Q}}^{\text {ext }}
$$

where $\mathbf{H}$ is the flow matrix and $\mathbf{Q}^{\text {ext }}$ is the fluid supply vector. See Appendix for details of the expression of the vectors and matrices in Equation (12).

\section{TIME-INTEGRATION}

Equations (10) and (12) are coupled to form the global system of equations in the following compact form

$$
\mathbf{D} \dot{\mathbf{X}}+\mathbf{K X}=\dot{\mathbf{W}}^{\text {ext }}
$$

where

$$
\mathbf{D}=\left[\begin{array}{cc}
\mathbf{K}^{e p} & \mathbf{L} \\
\mathbf{L}^{\mathrm{T}} & \mathbf{0}
\end{array}\right], \quad \mathbf{K}=\left[\begin{array}{cc}
\mathbf{0} & \mathbf{0} \\
\mathbf{0} & \dot{\mathbf{H}}
\end{array}\right], \quad \mathbf{X}=\left\{\begin{array}{l}
\mathbf{u} \\
\mathbf{p}
\end{array}\right\}, \quad \dot{\mathbf{W}}^{e x t}=\left\{\begin{array}{c}
\dot{\mathbf{F}}^{e x t} \\
\dot{\mathbf{Q}}^{e x t}
\end{array}\right\}
$$

Over the past decades several time-stepping schemes have been developed for solving Equation (13), see e.g. [4][11][21][23]. A popular method to integrate Equation (13) is the so called $\theta$-method. With this method, all time dependent variables are estimated at some intermediate point within the interval depending on the value of $\theta$. The $\theta$-method is usually used in an implicit form requiring iterations during each time step. Choosing $\theta=1$ leads to the classical backward Euler scheme, which is adopted in this study. Applying the method to Equation (13) provides 


$$
\mathbf{D}\left\{\mathbf{X}_{t+\Delta t}-\mathbf{X}_{t}\right\}+\Delta t \mathbf{K} \mathbf{X}_{t+\Delta t}=\Delta t \dot{\mathbf{W}}_{t+\Delta t}^{e x t}
$$

where $\Delta t$ is the step size, and $t$ and $t+\Delta t$ represent the quantities evaluated at the start and end of the increment, respectively. All variables except $\mathbf{X}_{t+\Delta t}$ in Equation (15) are known. In consolidation problems involving non-linear material behaviour $\mathbf{D}$ depends on $\mathbf{X}$, viz., Equation (15) must be solved by iteration. Applying the standard Newton-Raphson method, the residual vector is given by

$$
\begin{aligned}
\mathbf{R}\left(\mathbf{X}_{t+\Delta t}\right) & =\Delta t \dot{\mathbf{W}}_{t+\Delta t}^{e x t}-\mathbf{D}\left\{\mathbf{X}_{t+\Delta t}-\mathbf{X}_{t}\right\}-\Delta t \mathbf{K} \mathbf{X}_{t+\Delta t} \\
& =\left\{\begin{array}{c}
\Delta \mathbf{F}_{t+\Delta t}^{e x t} \\
\Delta \mathbf{Q}_{t+\Delta t}^{e x t}
\end{array}\right\}-\left[\begin{array}{cc}
\mathbf{K}^{e p} & \mathbf{L} \\
\mathbf{L}^{\mathrm{T}} & \mathbf{0}
\end{array}\right]\left\{\begin{array}{l}
\mathbf{U}_{t+\Delta t}-\mathbf{U}_{t} \\
\mathbf{P}_{t+\Delta t}-\mathbf{P}_{t}
\end{array}\right\}-\left[\begin{array}{cc}
\mathbf{0} & \mathbf{0} \\
\mathbf{0} & \Delta t \dot{\mathbf{H}}
\end{array}\right]\left\{\begin{array}{l}
\mathbf{U}_{t+\Delta t} \\
\mathbf{P}_{t+\Delta t}
\end{array}\right\} \\
& =\left\{\begin{array}{l}
\Delta \mathbf{F}_{t+\Delta t}^{e x t} \\
\Delta \mathbf{Q}_{t+\Delta t}^{e x t}
\end{array}\right\}-\left\{\begin{array}{l}
\Delta \mathbf{F}_{t+\Delta t}^{i n t} \\
\Delta \mathbf{Q}_{t+\Delta t}^{i n t}
\end{array}\right\}
\end{aligned}
$$

The iterative backward Euler updates are

$$
\delta \mathbf{X}_{j}=\left((\mathbf{D}+\Delta t \mathbf{K}) \mathbf{X}_{t+\Delta t}^{j-1}\right)^{-1} \mathbf{R}_{t+\Delta t}\left(\mathbf{X}_{t+\Delta t}^{j-1}\right)
$$

where $j$ denotes the iteration number. The iteration is terminated if

$$
\frac{\left\|\mathbf{F}_{t+\Delta t}^{e x t}-\mathbf{F}_{t+\Delta t}^{i n t}\right\|_{2}}{\left\|\mathbf{F}_{t+\Delta t}^{\text {ext }}\right\|_{2}} \leq \text { ITOL }
$$

where ITOL is a tolerance typically in the range $10^{-3}$ to $10^{-6}$ [11]. It is notable that Booker and Small [4] showed that the $\theta$-method is unconditionally stable provided $\theta \geq 0.5$. In another study, Vermeer and Verruijt [32] also confirmed this stability condition, and showed that for values of $\theta<0.5$ the minimum time step during consolidation is limited by

$$
t_{\min }=h^{2} \frac{\gamma_{w}}{6 E k}
$$

where $h$ is the distance between the Gauss points near the drainage surface, and $E$ represents the Young's modulus of the soil skeleton.

\section{MAXIMUM-ENTROPY FUNCTIONS}

The concept of maximum entropy (max-ent) originates from information theory where the notion of entropy was introduced as a measure of uncertainty [17]. According to this theory the entropy of a discrete probability distribution is 


$$
H(P)=-\sum_{i=1}^{n} P_{i} \ln P_{i}
$$

where $P_{i} \equiv P\left(x_{i}\right)$ is the probability of the occurrence of the event $x_{i}$ and $n$ represents the number of events. Jaynes [5] introduced the principle of maximum entropy as a means for finding the most likely probability distribution when insufficient information is available. According to this principle the discrete probabilities are obtained by solving the following constrained optimisation problem

$$
\begin{array}{ll}
\text { Maximise: } & H(P)=-\sum_{i=1}^{n} P_{i} \ln P_{i} \\
\text { subject to: } & \sum_{i=1}^{n} P_{i}=1, \quad \sum_{i=1}^{n} P_{i} g_{r}\left(x_{i}\right)=E\left(g_{r}(x)\right)
\end{array}
$$

where $E\left(g_{r}(x)\right)$ is the expectation of a function $g_{r}(x)$. Based on the principle of maximum entropy, and observing the analogy between the probabilities and the value of shape functions, Sukumar and Wright [26] proposed the max-ent shape functions by incorporating the weight functions, $w_{i}$, that provide a compact support. Following a similar fashion, the max-ent shape functions, $\Phi_{i}$, are obtained by solving

$$
\begin{array}{ll}
\text { Maximise: } & H(\Phi, w)=-\sum_{i=1}^{n} \Phi_{i} \ln \left(\frac{\Phi_{i}}{w_{i}}\right) \\
\text { subject to: } & \sum_{i=1}^{n} \Phi_{i}=1, \quad \sum_{i=1}^{n} \Phi_{i} x_{i}=x, \quad \sum_{i=1}^{n} \Phi_{i} y_{i}=y, \quad \sum_{i=1}^{n} \Phi_{i} z_{i}=z
\end{array}
$$

and are given as (see [26][27] for details)

$$
\Phi_{i}=\frac{w_{i} e^{-\lambda_{1}\left(x_{i}-x\right)-\lambda_{2}\left(y_{i}-y\right)-\lambda_{3}\left(z_{i}-z\right)}}{\sum_{j=1}^{n} w_{j} e^{-\lambda_{1}\left(x_{j}-x\right)-\lambda_{2}\left(y_{j}-y\right)-\lambda_{3}\left(z_{j}-z\right)}}
$$

where $\lambda_{1}, \lambda_{2}$, and $\lambda_{3}$ are the Lagrange multipliers associated with constraints in Equation (21), and implicitly depend on the position of point $x$.

The weight functions in the meshless methods are not unique [3]. In fact, the continuity of shape functions depend on the smoothness of the weight functions [8]. The weight functions must be positive within the support domain, zero outside the support domain, and should monotonically decrease from the point of interest while being sufficiently smooth near the boundary of a support domain (see Figure 1). In this study we adopt a cubic spline shape function which has $2^{\text {nd }}$ order continuity and is given by 


$$
w\left(r_{i}\right)= \begin{cases}\frac{2}{3}-4 r_{i}^{2}+4 r_{i}^{3} & \text { if } r_{i} \leq \frac{1}{2} \\ \frac{4}{3}-4 r_{i}+4 r_{i}^{2}-\frac{4}{3} r_{i}^{3} & \text { if } \frac{1}{2}<r_{i} \leq 1 \\ 0 & \text { if } r_{i}>1\end{cases}
$$

where $r_{i}$ is a normalised distance as defined by

$$
r_{i}=\frac{d_{i}}{d_{m i}}
$$

in which $d_{i}$ represents the distance from $i^{\text {th }}$ field node to the point of interest, and $d_{m i}$ is the influence domain of $i^{\text {th }}$ node. The size of the influence domain of a node is determined by

$$
d_{m i}=\alpha_{s} c_{i}
$$

where $\alpha_{s}$ is a scalar parameter and $c_{i}$ represents the distance between two adjacent nodes in uniformly distributed nodes. If the nodes are non-uniformly distributed, $c_{i}$ will be defined as an average nodal spacing in the domain of node $i$. Typical values of $\alpha_{s}$ are between 2.0 and 4.0 [8]. In this study, a circular support domain for two-dimensional problems is used, see Figure 1.

\section{NUMERICAL EXAMPLES}

The numerical method explained in the previous sections was implemented into a computer program. This code is used for analysing all numerical examples presented in this section. These problems include one-dimensional consolidation of an elastic soil layer, two-dimensional consolidation of an elastic soil layer beneath a strip footing, elasto-plastic consolidation of a soil layer supporting a strip and circular footing under uniaxial vertical load, and the undrained bearing capacity of soil under a strip footing.

The MEM method is validated by comparing its results with FE results or with closed-form analytical solutions available in the literature. The FE results are obtained by SNAC, an in-house finite element analysis developed by the geotechnical group at the University of Newcastle, which has been extensively validated and used to analyse geotechnical problems [11][23][24].

\section{One-dimensional elastic consolidation analysis}

Terzaghi [29] presented an analytical solution for the one-dimensional consolidation of an elastic soil layer subjected to a uniform surface pressure. The problem domain and discretisation by the MEM method, boundary conditions, and material properties are shown in Figure 2.a. Note that only a onedimensional soil column with unit width is considered in the analysis, which is discretised by 63 field nodes. The scaling parameter $a_{s}$ is assumed to be 3.0, number of Gauss points in $1 \mathrm{D}$ is 3 , and the background points are the same as field nodes. In Figure 2.a, $E^{\prime}, v^{\prime}, k$, and $\gamma_{w}$ represent the drained 
Young's modulus, drained Poisson's ratio, coefficient of isotropic permeability of the soil, and the unit weight of water, respectively.

According to the analytical solution, the excess pore water pressure, $p$, dissipates over the dimensionless time, $T_{v}$, according to

$$
p(y, t)=2 q \sum_{n=0}^{\infty} \frac{1}{\alpha_{n}} \sin \alpha_{n} \frac{y}{H} e^{-\alpha_{n}^{2} T_{v}}, \alpha_{n}=(2 n+1) \frac{\pi}{2}
$$

where $q$ is the surface surcharge, $H$ represent the drainage length, and $T_{v}$ is the dimensionless time parameter

$$
T_{v}=\frac{c_{v} t}{H^{2}}
$$

in which $c_{v}$ denotes the coefficient of consolidation of soil as given by

$$
c_{v}=\frac{k E^{\prime}\left(1-v^{\prime}\right)}{\gamma_{w}\left(1+v^{\prime}\right)\left(1-2 v^{\prime}\right)}
$$

In the analytical solution, it is assumed that the surcharge $q$ is applied on the soil instantaneously. In a numerical analysis, this boundary condition is usually satisfied by applying the surcharge over a relatively short period of time. In this example the analysis is performed in two stages. In first stage, the surcharge is applied on top of a soil column over a period of $T_{v}=10^{-4}$, assuring a loading rate fast enough to avoid dissipation of excess pore water pressures. Next, the load is kept constant and the soil is allowed to consolidate over a long period of time $T_{v}=3$. The normalised excess pore water pressure (at $y=H$ ), predicted by the MEM method and Equation (26), is plotted versus $T_{v}$ in Figure 2b. According to Figure $2 \mathrm{~b}$, there is a good agreement between the numerical MEM results and the analytical solution. In a previous study, Shibata and Murakami [22] showed that numerical instabilities are usually encountered in the analysis of one-dimensional consolidation using the EFG method based on the MLS shape functions unless a stabilisation technique is employed. Such numerical instabilities generally lead to oscillation in the excess pore water pressure throughout the soil column regardless of the nodal distribution (see [22] for more details). It is notable that the numerical MEM solution based on the max-ent shape functions is stable and there is no oscillation in the predicted excess pore water pressure.

\section{Two-dimensional elastic consolidation analysis}

The second example involves the consolidation of an elastic and weightless soil layer under a flexible strip footing. The problem geometry, boundary conditions, and material properties are shown in Figure 3.a. Note that the loaded area under the footing is assumed to be permeable. The problem is solved by the FE method as well as the MEM method. Due to symmetry only half of the problem domain is considered, and plane strain conditions are assumed. The FE mesh, including 288 6-noded triangular elements and 625 nodal points, is shown in Figure 3.b. In comparison the MEM discretisations by a 
uniform mesh, including 441 field nodes and 400 background cells, as well as a non-uniform mesh, containing 589 field nodes and 400 background cells, are depicted in Figure 3.c and Figure 3.d, respectively. Parameter $\alpha_{s}$ is set to 3.0 and the number of Gauss points in one direction is assumed to be 4.

In all analyses, the uniform pressure is applied on the soil over a period of $T_{v}=5 \times 10^{-5}$, followed by a consolidation phase over a period of $T_{v}=12$. The settlement of a node on the centreline of the footing, normalised by the footing width and the ratio between the shear modulus of soil $(G)$ and the applied pressure, from the FE method as well as the MEM method, is plotted versus $T_{v}$ in Figure 4.d. The settlements predicted by the two methods are essentially identical, validating the accuracy as well as the stability of the proposed meshless method.

\section{Two-dimensional elasto-plastic consolidation analysis}

In the third example, we consider a more complex problem including two-dimensional consolidation of an elasto-plastic soil layer under a strip as well as a circular footing. Plane strain and axisymmetric conditions are assumed for the strip and the circular footings, respectively. In all analyses presented in this example, the behaviour of soil is modelled using a rounded Mohr-Coulomb constitutive model proposed by Sheng et al. [20] that uses a non-associated flow rule. In this model the yield function, $f$, and the plastic potential, $g$, are defined by

$$
\begin{aligned}
& f=q-\frac{6 \sin \phi^{\prime}}{3-\sin \phi^{\prime}}\left(\frac{c^{\prime}}{\tan \phi^{\prime}}+p^{\prime}\right)\left(\frac{2 \alpha^{4}}{1+\alpha^{4}-\left(1-\alpha^{4}\right) \sin 3 \theta_{l}}\right)^{1 / 4}, \quad \alpha=\frac{3-\sin \phi^{\prime}}{3+\sin \phi^{\prime}} \\
& g=q-\frac{6 \sin \psi^{\prime}}{3-\sin \psi^{\prime}}\left(\frac{c^{\prime}}{\tan \psi^{\prime}}+p^{\prime}\right)\left(\frac{2 \alpha^{4}}{1+\alpha^{4}-\left(1-\alpha^{4}\right) \sin 3 \theta_{l}}\right)^{1 / 4}
\end{aligned}
$$

where $q$ is the deviatoric stress, $p^{\prime}$ represents the effective mean stress, $c^{\prime}, \phi^{\prime}$, and $\psi^{\prime}$ are the drained cohesion, friction angle, and dilation angle of soil, respectively, and $\theta_{l}$ denotes the Lode angle. Due to the nonlinear behaviour of soil, the differential equation in (6) is solved by an explicit stress-integration scheme proposed in [24][12].

The problem domain, material properties, and boundary conditions are shown in Figure 5. Only half of the problem domain is considered in all analyses. In Figure 5, B represents the total width of a strip footing as well as the diameter of a circular footing, and $\gamma$ is the unit weight of soil. The problem is solved by the FE and the MEM methods and the numerical results are compared with each other. In the FE analyses, four regular meshes discretised by 8-noded rectangular elements, as shown in Figure 6, were used. The main purpose of using different meshes was to demonstrate the effect of mesh density on the numerical results. The problem domain was also discretised by four different MEM grids, as depicted in Figure 7. Similar to FE mesh, the field nodes in MEM grids are distributed in an irregular fashion over 
the problem domain. The value of $\alpha_{s}$ is assumed to be 2.0 in all meshless analyses. The topology information of FE meshes and MEM grids are summarised in Table 1.

The consolidation analysis in this example includes three stages. In stage 1, body force loading due to the self-weight of the soil and water is applied to generate a non-zero initial stress field and a hydrostatic pore water pressure profile in the soil. In the second stage, a uniform pressure is applied on soil over a period of $T_{v}=1.35 \times 10^{-5}$, using 100 equal time increments. The magnitude of applied pressures in the plane strain analysis and the axisymmetric analysis are $4 c^{\prime}$ and $5 c^{\prime}$, respectively, ensuring that the soil behaviour is elasto-plastic. As noted by Small [28], it is important to assume a zero value for the dilation angle since the soil deforms in an undrained condition during loading. Small [28] observed that a coupled consolidation analysis with an elasto-plastic constitutive model, such as Mohr-Coulomb, is unable to accurately model the undrained behaviour unless a zero dilation angle is used. This is due to the fact that a non-zero dilation angle causes a drop in the excess pore pressure upon plastic shearing, leading to an unrealistic (artificial) hardening in the soil response. In the third stage of analysis, the uniform pressure is kept constant and the soil is allowed to consolidate over a long period, $T_{v}=15$, ensuring that excess pore water pressures fully dissipate.

The settlement of a point at the centre of the footing, normalised by $B$ and multiplied by the ratio between the shear modulus of soil and its drained cohesion, is plotted versus $T_{v}$ in Figure 8. Figure 8.a and Figure 8.b shows the settlement of the strip footing as predicted by the FE method and the MEM method, respectively, indicating good agreement between the two methods, irrespective of the mesh density. For the circular footing, the FE results and the MEM results are shown in Figure 8.c and Figure 8.d, respectively. In this case, the difference between the numerical results is significant. Analyses based on the coarser FE meshes, A and B, predict different and lower settlements than the finer meshes, C and D, which are seen to converge (albeit mesh B predicts only slightly lower settlements than its finer counterparts, C \& D). MEM Grids 1 and 2 overestimate the settlements predicted by MEM Grids 3 and 4, though the sensitivity to grid density is much less than that observed for mesh size in the traditional finite element approach. In overall, there is a good agreement between the numerical results obtained by the FE and the MEM methods. The values of immediate settlements as well as the final settlements normalised by $B$ are summarised in Table 1.

The contours of excess pore water pressure developed at the end of loading phase, obtained by MEM Grid 2 as well as MEM Mesh D, are depicted in Figure 9. It is observed that the solution obtained by the MEM method is stable.

To compare the performance of MEM method and the FE method, the normalised CPU time and the error predicted by each analysis are presented in Table 2. The normalised CPU time is calculated by diving the analysis time by the time of fastest analysis. For the FE meshes, the error is obtained by finding the difference between the final settlement predicted by the analysis and the settlement predicted by Mesh D, 
assuming that Mesh D provides the most accurate answer among the FE meshes. On the other hand, the error of each MEM grid is computed according to the difference between the predicted final settlement and the settlement obtained by Grid 4. Table 2 also represents the number of active degrees-of-freedom in each analysis, being the difference between the total degrees-of-freedom (total number of nodes multiplied by 3) and the restrained degrees-of-freedom due to boundary conditions. The results are presented for the strip footing as well as the circular footing. The numerical values in Table 2 are graphically represented in Figure 10 by plotting the error versus the active degrees-of-freedom as well as the error versus the normalised CPU time. According to Figure 10.a and Figure 10.c the MEM method, compared with the FE method, can predict more accurate results with less number of degrees-of-freedom. For the plane strain analyses in Figure 10.b it is fair to say that the MEM method outperforms the FE method, in terms of computational time. It is clearly observed that the MEM method can achieve same accuracy as the FE method, but a lower computational time. This outperformance, however, is not significant for axisymmetric results presented in Figure 10.d, where both methods predict more or less same numerical results at approximately equal computational times.

It is notable that under the condition of identical degrees-of-freedom the MEM method is more accurate than the FE method (Figure 10.a and Figure 10.c), but fails to outperform the FE method for computational time. This is demonstrated in Figure 11 by plotting the normalised CPU time versus the active degrees-of-freedom for the plane strain as well as the axi-symmetric analyses. One main reason for this difference in computational time is that the band-width of matrix D (see Equation (13)), and consequently the number of non-zero terms, in the MEM method is usually larger than its counterpart in the FE method, leading to a more costly solution of global equations in each iteration. Generally speaking, a fair comparison between the Finite Element method and the Maximum-Entropy Meshless method may not be so straightforward due to the number of parameters involved in the solution. Although briefly presented in this example, the performance of the MEM method compared with other numerical methods, particularly in geotechnical problems, is not the main subject of this paper and requires further research.

\section{Undrained bearing capacity of a soil layer under a strip footing}

In this example, the ability of the proposed method to correctly predict coupled stress-pore fluid response is demonstrated for a complex problem involving the undrained bearing capacity of a uniform soil layer under plane strain conditions. In the literature, this problem has proved a robust benchmark for testing and validating numerical methods for analysing saturated porous media [1][23]. The problem follows the work of Small [28] who proved that a for a weightless soil the drained and undrained strength parameters are dependent according to 


$$
\frac{c_{u}}{c^{\prime}}=\frac{2 \sqrt{N_{\phi}}}{1+N_{\phi}}, \quad N_{\phi}=\frac{1+\sin \phi^{\prime}}{1-\sin \phi^{\prime}}
$$

where $c_{u}$ represents the undrained shear strength of soil.

The problem domain, boundary conditions, and soil properties are shown in Figure 12. The undrained bearing capacity of soil, $q_{u}$, is given by the well-known Prandtl formula

$$
q_{u}=(2+\pi) c_{u}=5.14 c_{u}
$$

In principle, a coupled analysis using the drained material properties must be able to predict the undrained failure load provided the loading is applied sufficiently fast to avoid the dissipation of excess pore water pressures. The problem domain is discretised by a uniform grid as well as a non-uniform grid, as shown in Figure 12.b and Figure 12.c, respectively. Note that the number of background cells in both grids is 400. The uniform load is applied on the soil at the rate of $\omega$, defined by

$$
\omega=\frac{4 B^{2}}{\Delta t c_{v}} \frac{\Delta q}{c^{\prime}}
$$

A rapid loading rate of $\omega=150$ is used to simulate the undrained conditions [17]. The undrained response of soil predicted by two MEM grids is shown in Figure 13. The ultimate load predicted by the uniform grid is $\sim 16 \%$ higher than the analytical solution, whereas the non-uniform grid is able to accurately predict the collapse load. This accurate prediction demonstrates the stability of the MEM method based on principle of maximum entropy for consolidation analysis of porous media.

\section{CONCLUSIONS}

A stable numerical procedure for analysing the consolidation problems of porous media within the framework of the Maximum-Entropy Meshless method has been presented in this study. The method takes advantage of the maximum entropy functions, and thus the essential boundary conditions are automatically satisfied and do not require any computational challenge. The proposed method was employed to solve several numerical examples including one-dimensional as well as two-dimensional consolidation of an elastic soil layer, elasto-plastic consolidation of a soil layer under a strip and a circular footing, and the undrained bearing capacity of a soil layer under plane strain conditions. Based on the numerical results presented here it is concluded that the max-ent shape functions provide an intrinsically stable means for solving elasto-plastic consolidation problems of porous media. Perhaps the main reason for such a robust performance is that max-ent shape functions are relatively insensitive to the distribution of unknown variables. Nonetheless, the mathematical proof demonstrating the stability of MaximumEntropy Meshless method for consolidation problems has not been given here, and appears to be extremely difficult to obtain. 
More importantly, it is concluded that the same shape functions can be used for interpolating displacements field and pore water pressures field. The numerical results also indicate that the method can provide accurate results provided an appropriate grid density is used in the analysis.

\section{ACKNOWLEDGEMENT}

This work forms part of the activities of the Australian Research Centre (ARC) Centre of Excellence for Geotechnical Science and Engineering. The authors are grateful for this ARC support. 


\section{REFERENCES}

[1] Abbo AJ (1997). Finite element algorithms for elastoplasticity and consolidation, PhD Thesis, The University of Newcastle, Australia.

[2] Arroyo M and Ortiz M (2006). Local maximum-entropy approximation schemes: a seamless bridge between finite elements and meshfree methods, International Journal for Numerical Methods in Engineering, 65(13):2167-2202.

[3] Belytschko T, Lu YY, and Gu L (1994). Element-free Galerkin methods, International Journal for Numerical Methods in Engineering, 3, 229-256.

[4] Booker JR and Small JC (1975). An investigation of the stability of numerical solutions of Biot's equations of consolidation, International Journal of Solids and Structures, 11, 907-917.

[5] Jaynes ET (1957). Information theory and statistical mechanics. Physical Review, 106(4), 620-630.

[6] Khoshghalb A (2011). Meshfree analysis of unsaturated porous media including hydraulic hysteresis and large deformations, PhD Thesis, The University of New South Wales, Sydney, Australia.

[7] Khoshghalb A, and Khalili N (2010). A stable meshfree method for fully coupled flow deformation analysis of saturated porous media. Computers and Geotechnics, 37, 789-795.

[8] Liu GR and Gu YT (2005). An introduction to meshfree methods and their programming, Springer, The Netherlands.

[9] Millan D, Rosolen A, and Arroyo M (2011). Thin shell analysis from scattered points with maximumentropy approximants. International Journal for Numerical Methods in Engineering, 85(6), 723-751.

[10] Modaressi H and Aubert P (1998). Element-free Galerkin method for deforming multiphase porous media, International Journal for Numerical Methods in Engineering, 42, 313-340.

[11] Nazem M, Sheng D, Carter JP and Sloan SW (2008). Arbitrary-Lagrangian-Eulerian method for large-deformation consolidation problems in geomechanics. International Journal of Numerical and Analytical Methods in Geomechanics, 32, 1023-1050.

[12] Nazem M, Carter JP, Sheng D, and Sloan SW (2009). Alternative stress-integration schemes for large-deformation problems of solid mechanics, Finite Elements in Analysis and Design, 45(12), 934943.

[13] Nogami T, Wang W, and Wang JG (2004). Numerical method for consolidation analysis of lumpy clay fillings with meshless method, Soils and Foundations, 44, 125-142.

[14] Oliaei MN, Soga K, and Pak A (2009). Some numerical issues using element-free Galerkin meshless method for coupled hydro-mechanical problems, International Journal of Numerical and Analytical Methods in Geomechanics, 33, 915-938.

[15] Ortiz A, Puso M, and Sukumar N (2011). Maximum-entropy meshfree method for incompressible media problems, Finite Elements in Analysis and Design, 47(6), 572 - 585.

[16] Potts DM and Zdravkovicz L (1999). Finite element analysis in geotechnical engineering: Theory, Thomas Telford, London.

[17] Shannon CE (1948). A mathematical theory of communication. The Bell Systems Technical Journal, 27, 379-423.

[18] Quaranta G, Kunnath S, and Sukumar N (2012). Maximum-entropy meshfree method for nonlinear static analysis of planar reinforced concrete structures, Engineering Structures 42,179-189.

[19] Samimi S, and Pak A (2012). Three-dimensional simulation of fully coupled hydro-mechanical behaviour of saturated porous media using Element Free Galerkin (EFG) method, Computers and Structures, 46, 75-83.

[20] Sheng D, Sloan SW, and Yu HS (2000). Aspects of finite element implementation of critical state models, Computational Mechanics, 26, 185-196.

[21] Sheng D and Sloan SW (2003). Time stepping schemes for coupled displacement and pore pressure analysis, Computational Mechanics, 31, 122-134. 
[22] Shibata T, and Murakami A (2011). A stabilization procedure for soil-water coupled problems using the element-free Galerkin method. Computers and Geotechnics, 38, 585-597.

[23] Sloan SW and Abbo AJ (1999). Biot consolidation analysis with automatic time stepping and error control, part 1: theory and implementation, International Journal for Numerical and Analytical Methods in Geomechanics, 23, 467-492.

[24] Sloan SW, Abbo AJ, and Sheng D (2001). Refined explicit integration of elastoplastic models with automatic error control, Engineering Computations, 18, 121-154.

[25] Sukumar N (2004) Construction of polygonal interpolants: a maximum entropy approach, International Journal for Numerical Methods in Engineering, 61, 2159-2181.

[26] Sukumar N and Wright WR (2007). Overview and construction of meshfree basis functions: From moving least squares to entropy approximation, International Journal for Numerical Methods in Engineering, 70, 181-205.

[27] Sukumar N (2008). Fortran 90 Library for Maximum-Entropy Basis Functions. User’s Reference Manual Version 1.4., Code available at http://www.imechanica.org/node/3424.

[28] Small JC (1977). Elasto-plastic consolidation of soils, PhD Thesis, University of Sydney, Australia.

[29] Terzaghi K (1960), From theory to practice in soil mechanics, John Wiley and Sons, New York, 133-146.

[30] Ullah Z and Augarde CE (2013). Finite deformation elasto-plastic modelling using an adaptive meshless method, Computers and Structures, 118, 39-52.

[31] Ullah Z, Coombs WM, and Augarde CE (2013). An adaptive finite element/meshless coupled method based on local maximum entropy shape functions for linear and nonlinear problems. Computer Methods in Applied Mechanics and Engineering, 267, 11-132.

[32] Vermeer PA and Verruijt A (1981). An accuracy condition for consolidation by finite elements, International Journal for Numerical and Analytical Methods in Geomechanics, 1, 1-4.

[33] Wang JG, Karim MR, and Lin PZ (2007). Analysis of seabed instability using element free Galerkin method, Ocean Engineering, 34, 247-260.

[34] Wang JG, Liu GR, and Lin PZ (2002). Numerical analysis of Biot's consolidation process by radial point interpolation method. International Journal for Solids and Structures, 39(6), 1557-1573.

[35] Wang JG, Yan L, and Liu GR (2005). A local radial point interpolation method for dissipation process of excess pore water pressure. International Journal for Numerical Methods in Heat and Fluid Flow, 15(6), 567-587. 


\section{Appendix}

The stiffness matrix, coupling matrix, and the vector of internal nodal forces in Equation (10) are defined by

$$
\begin{aligned}
& \mathbf{K}^{e p}=\int_{\Omega} \mathbf{B}_{u}^{T} \mathbf{D}^{\prime e p} \mathbf{B}_{u} d \Omega \\
& \mathbf{L}=\int_{\Omega} \mathbf{B}_{u}^{T} \mathbf{m} \boldsymbol{\Phi} d \Omega \\
& \mathbf{F}^{i n t}=\int_{\Omega} \mathbf{B}_{u}^{T} \boldsymbol{\sigma}^{\prime} d \Omega+\int_{\Omega} \mathbf{B}_{u}^{T} \mathbf{m p} d \Omega
\end{aligned}
$$

The flow matrix and the fluid supply vector in Equation (12) are defined by

$$
\begin{aligned}
\dot{\mathbf{H}} & =\frac{1}{\gamma_{w}} \int_{\Omega} \mathbf{B}_{p}^{T} \mathbf{k B}_{p} d \Omega \\
\dot{\mathbf{Q}} & =-\int_{\Gamma_{q}} \boldsymbol{\Phi q} d \Gamma_{q}-\frac{1}{\gamma_{w}} \int_{\Omega} \mathbf{B}_{p}^{T} \mathbf{k} \mathbf{b}_{w} d \Omega
\end{aligned}
$$

For two-dimensional plane strain problems, the corresponding $\mathbf{B}$ matrices at $i^{\text {th }}$ field node located inside the support domain of a Gauss point is defined by

$$
\begin{aligned}
\mathbf{B}_{u} & =\left[\begin{array}{lll}
\frac{\partial \Phi_{i}}{\partial x} & 0 & \frac{\partial \Phi_{i}}{\partial y} \\
0 & \frac{\partial \Phi_{i}}{\partial y} & \frac{\partial \Phi_{i}}{\partial x}
\end{array}\right]^{T} \\
\mathbf{B}_{p} & =\left[\begin{array}{c}
\frac{\partial \Phi_{i}}{\partial x} \\
\frac{\partial \Phi_{i}}{\partial y}
\end{array}\right]
\end{aligned}
$$

For plane strain conditions, $\mathbf{m}$ and $\boldsymbol{\sigma}^{\prime}$ are given by

$$
\begin{aligned}
& \mathbf{m}=\left\{\begin{array}{lll}
1 & 1 & 0
\end{array}\right\}^{T} \\
& \boldsymbol{\sigma}^{\prime}=\left\{\begin{array}{lll}
\sigma_{x x} & \sigma_{y y} & \sigma_{x y}
\end{array}\right\}^{T}
\end{aligned}
$$




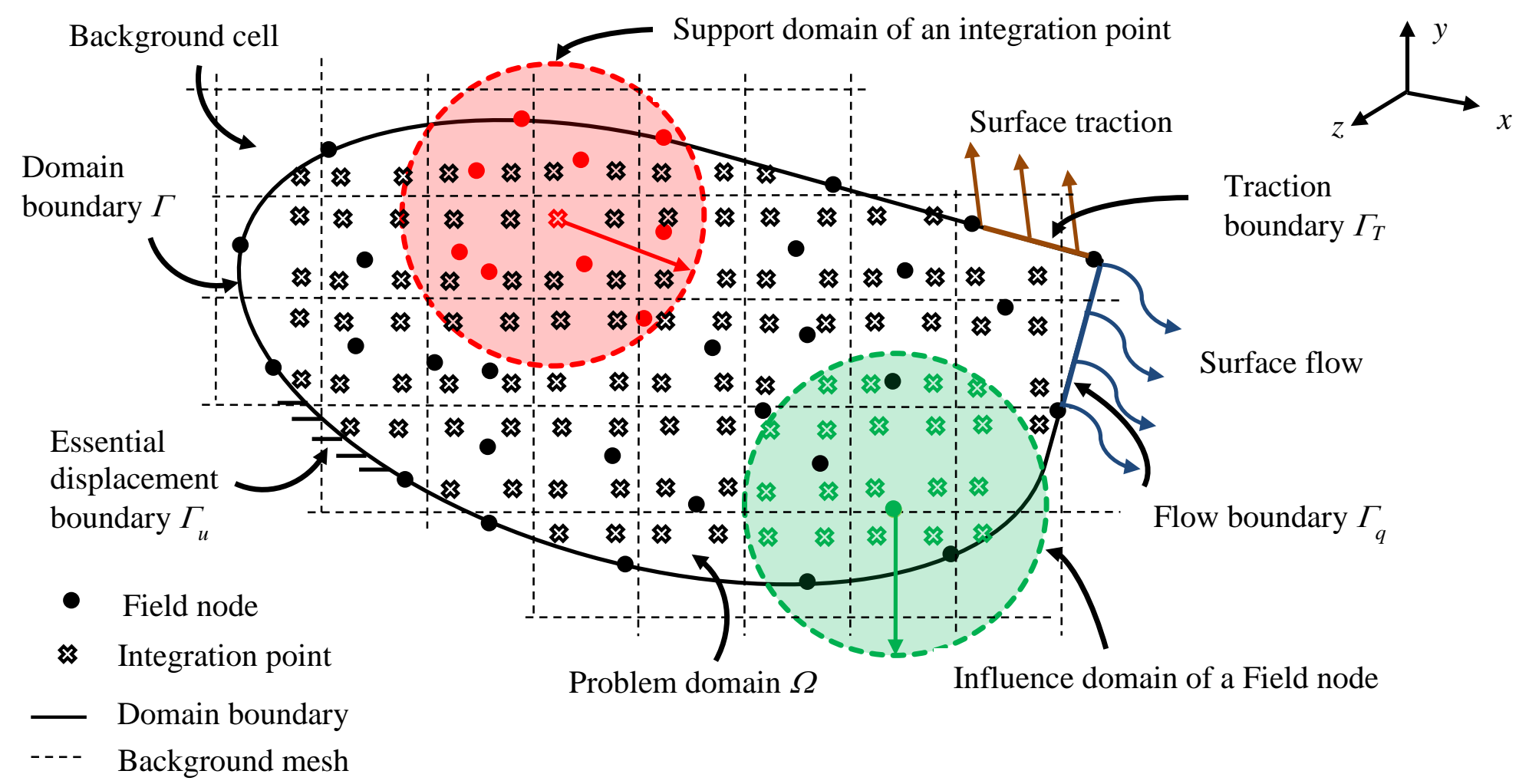

Figure 1 . Problem discretisation by the MEM method. 


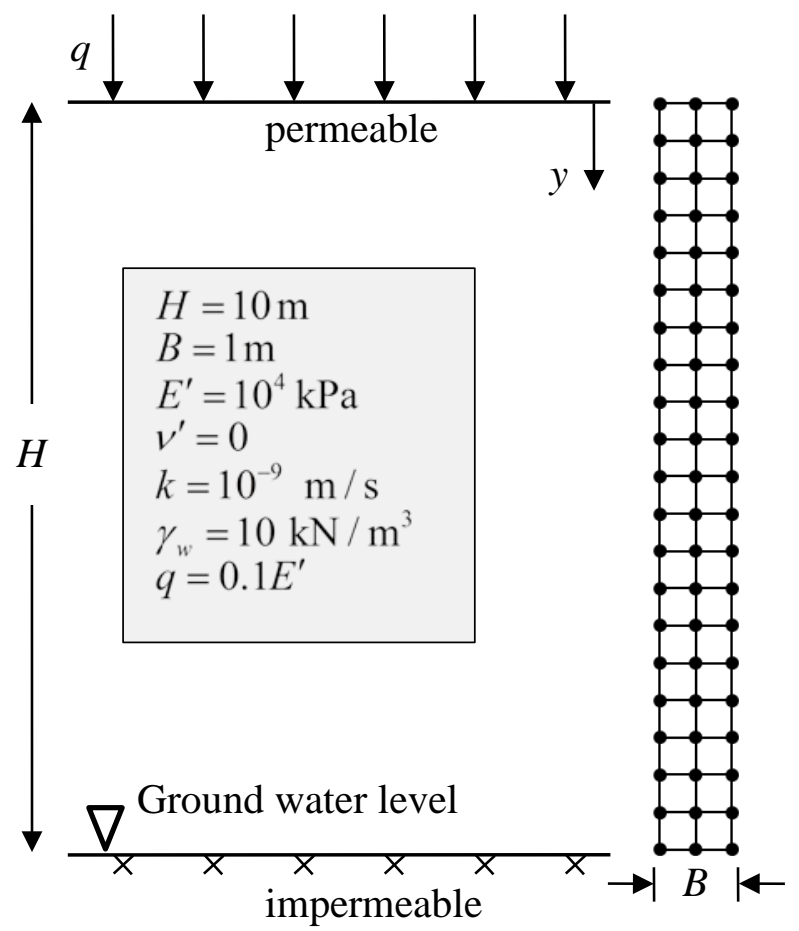

a. Problem definition and domain discretisation.

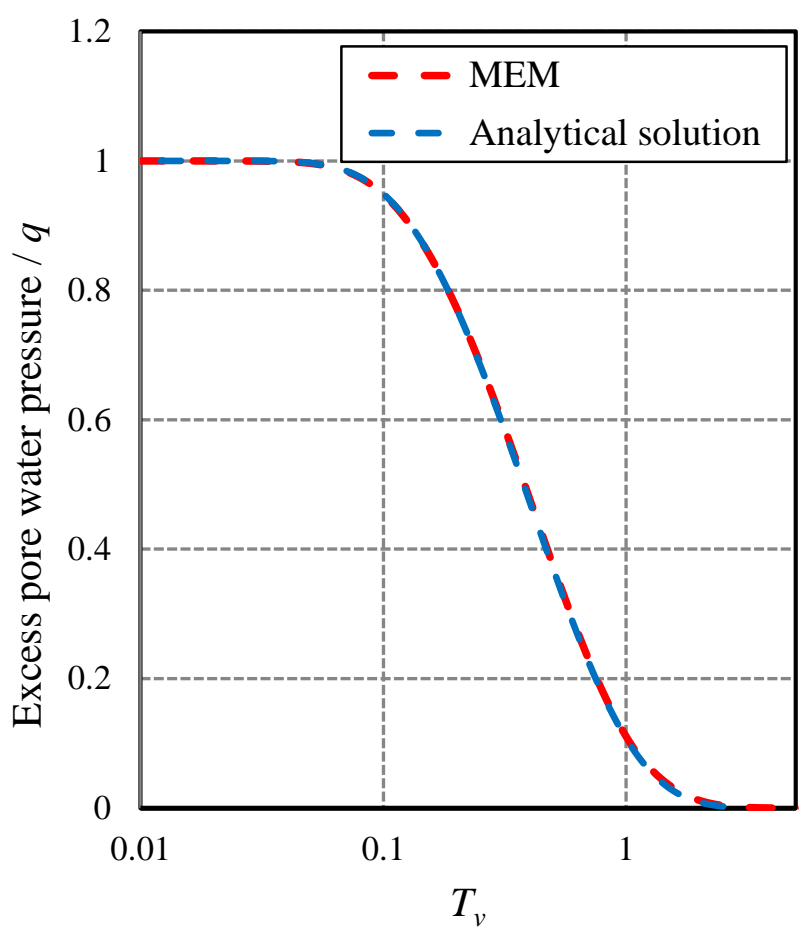

b. Dissipation of excess pore pressure.

Figure 2. One-dimensional consolidation of an elastic soil layer. 


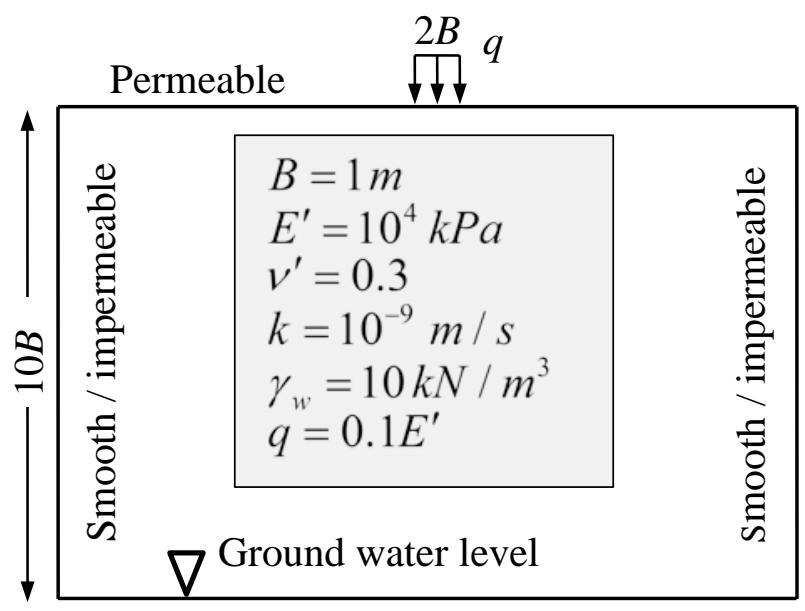

Rough / impermeable

$$
20 B
$$

a. Problem domain, boundary conditions, and material properties.

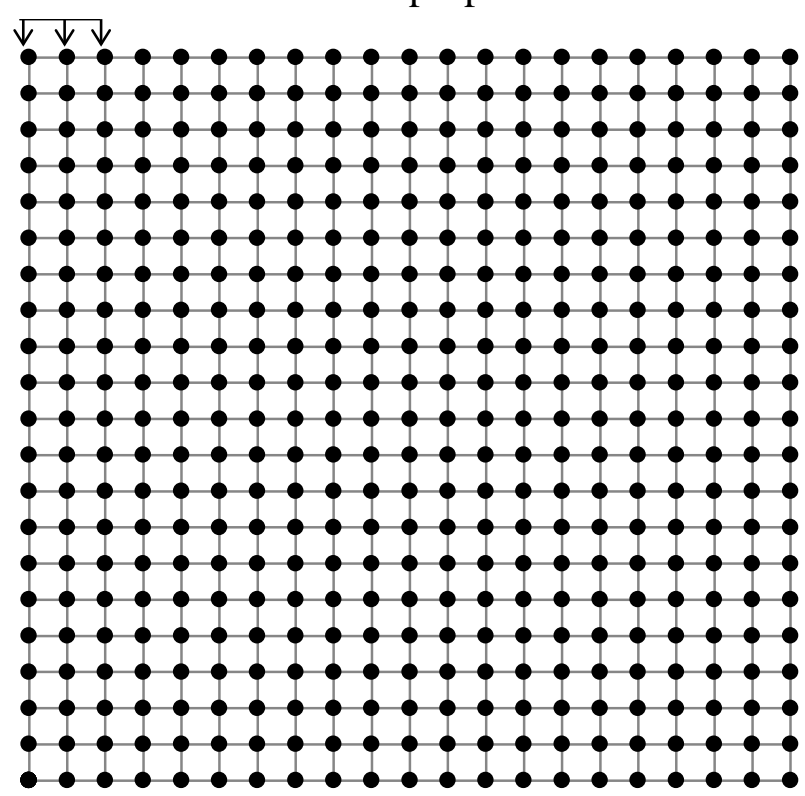

c. Uniform MEM discretisation, 441 field nodes, 400 background cells.

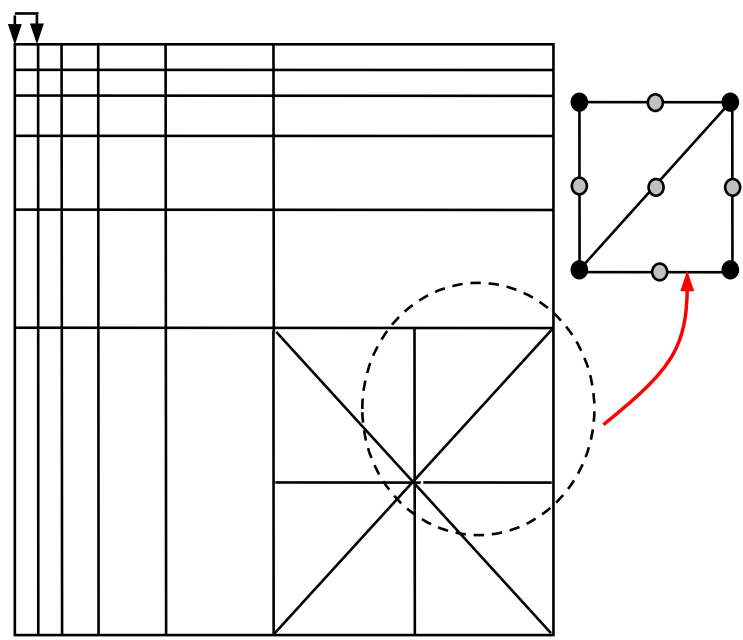

○ Displacement nodes

- Displacement and pore pressure nodes

b. FE discretisation, 288 6-node triangular elements and 625 nodal points.

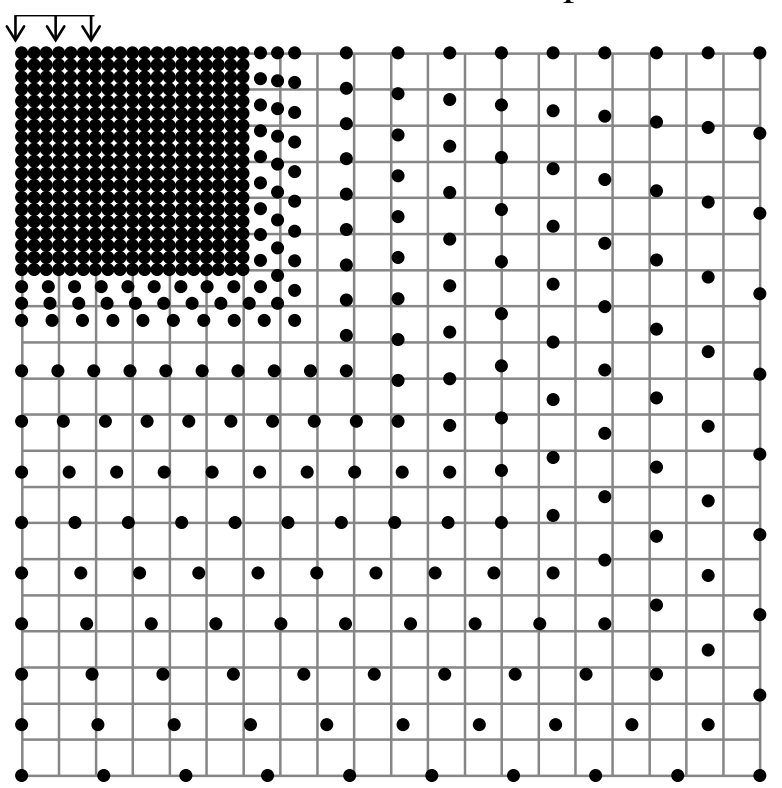

d. Non-uniform MEM discretisation, 589 field nodes, 400 background cells.

Figure 3. Flexible rough footing on an elastic layer of soil 


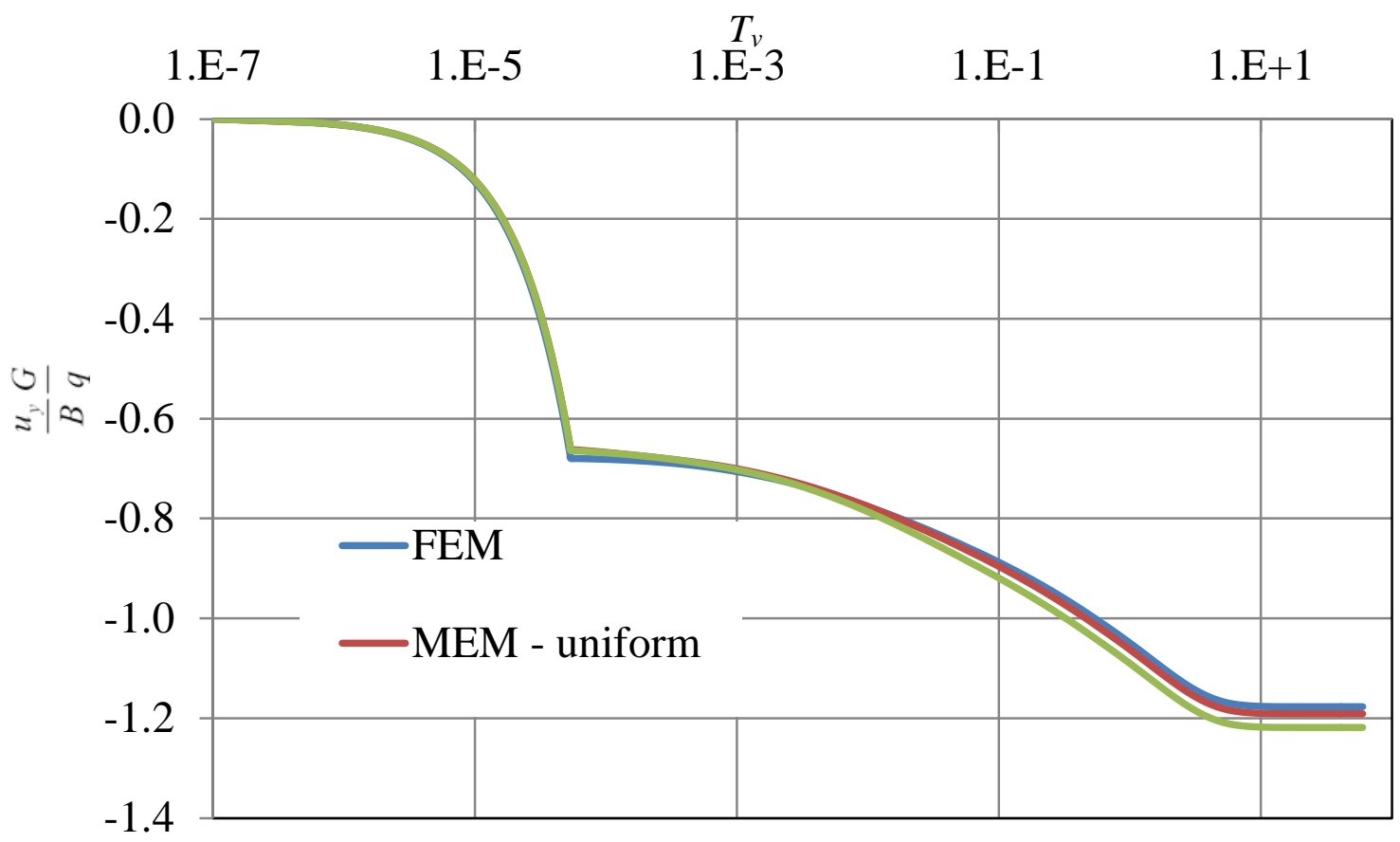

Figure 4. Normalised vertical settlement of footing centre versus $T_{V}$ 


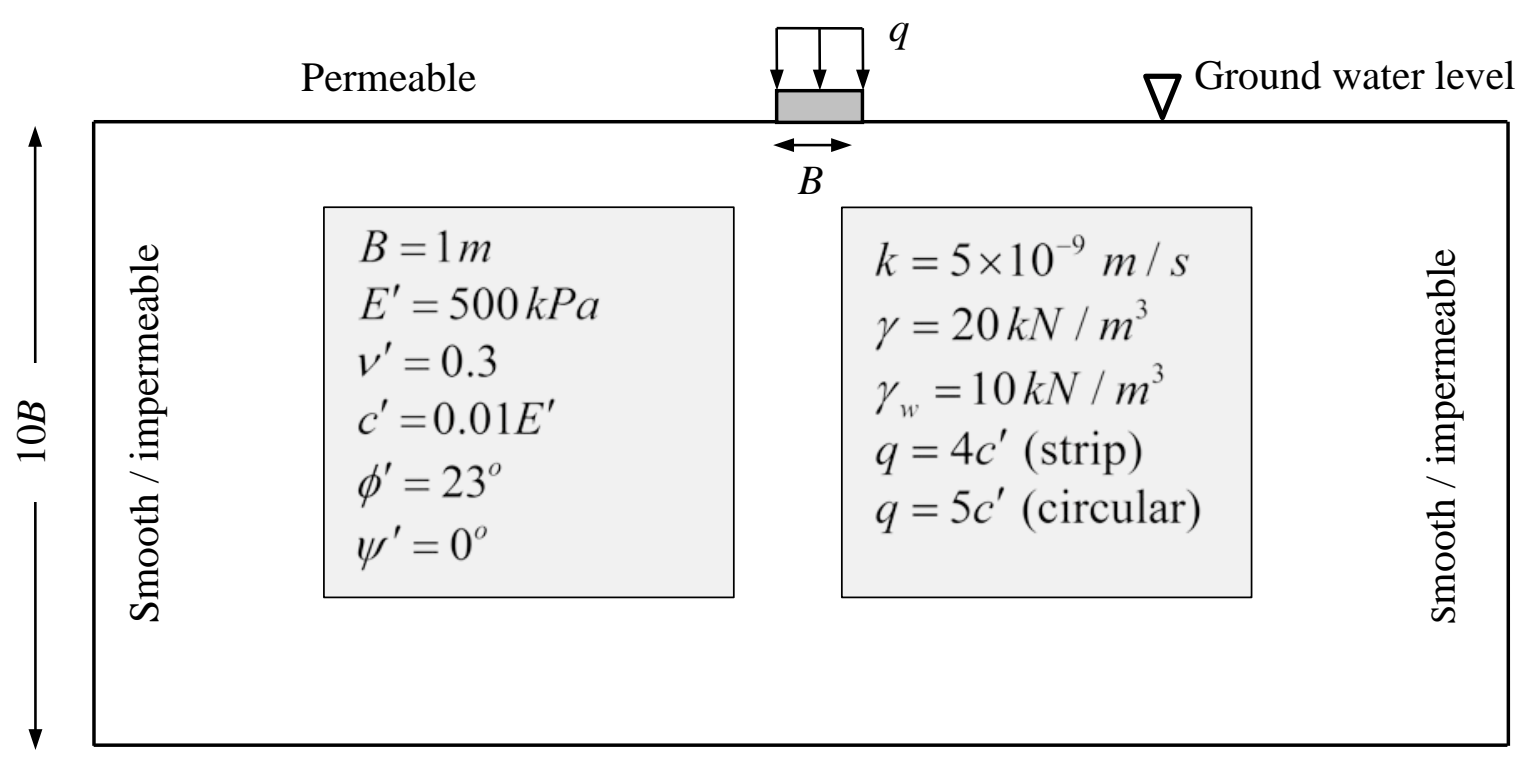

Rough / impermeable

$20 B$

Figure 5. Elastoplastic consolidation of a soil layer under a flexible rough strip/circular footing. 


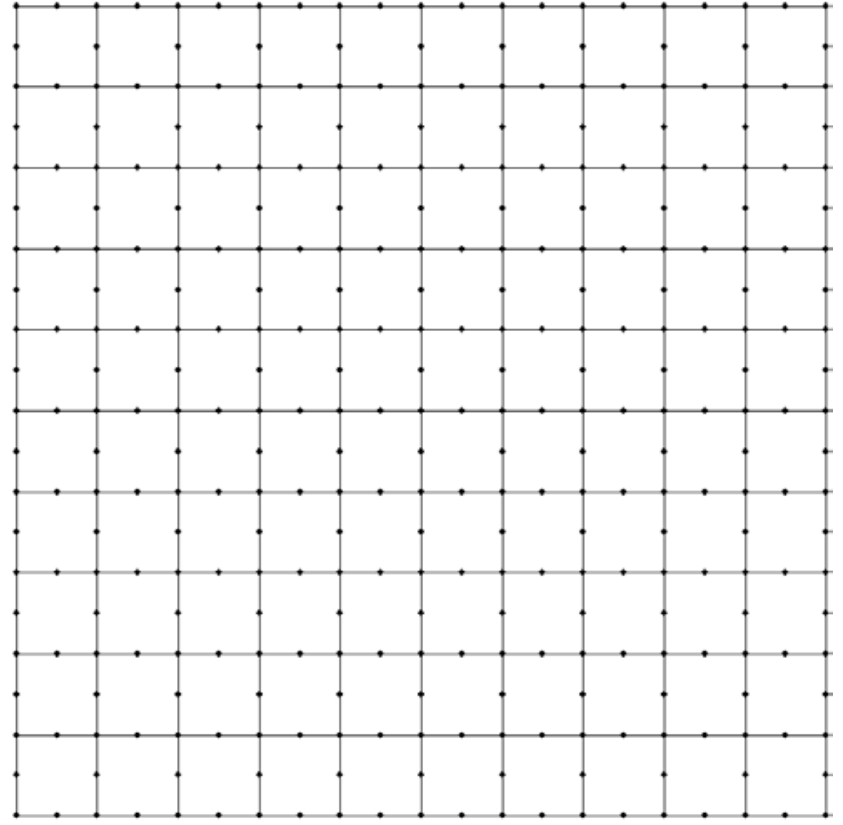

a. FE mesh A.

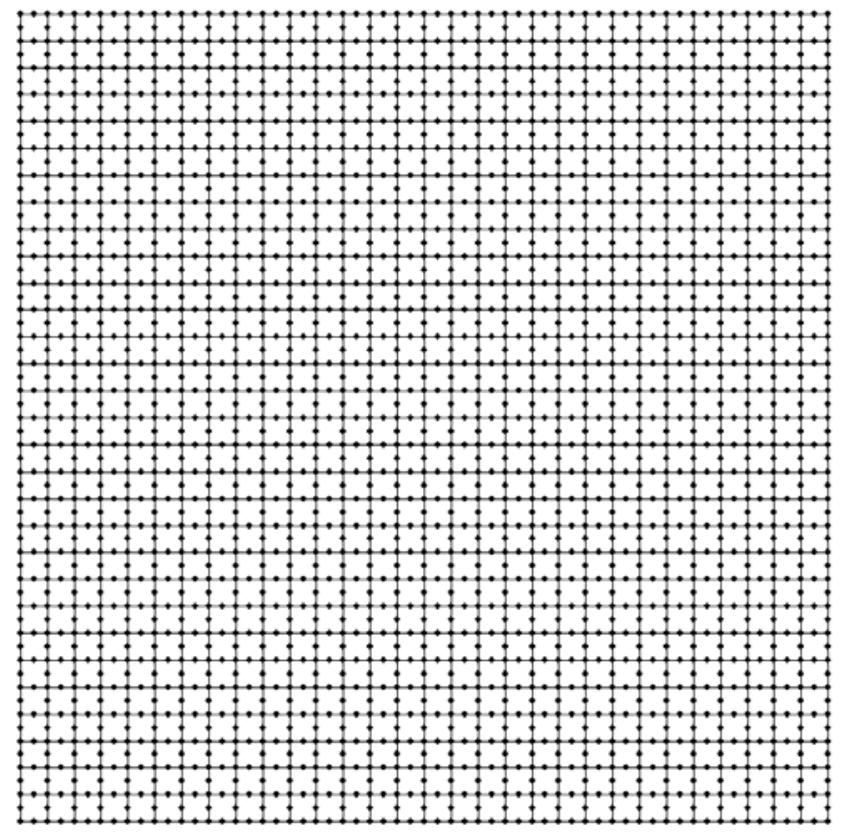

c. FE mesh C.

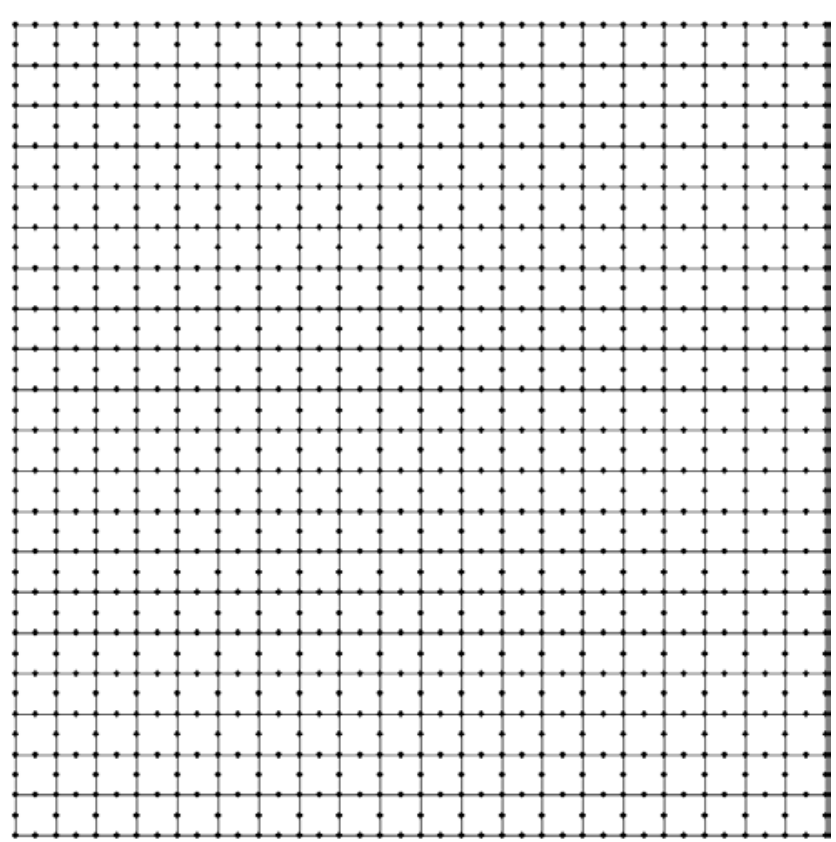

b. FE mesh B.

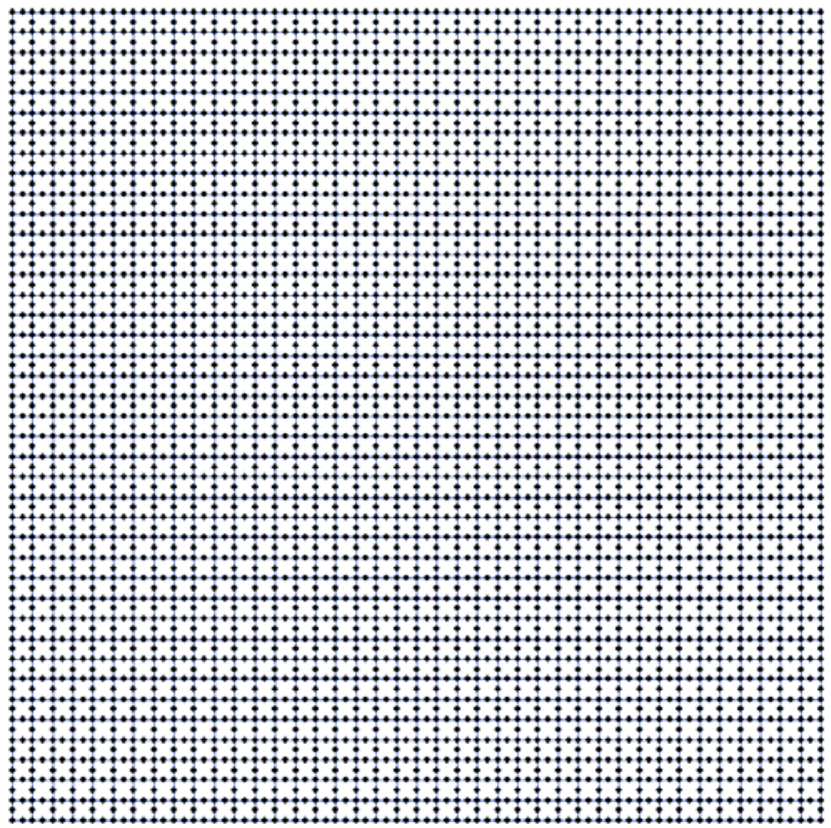

d. FE mesh D.

Figure 6. FE meshes used for analysing two-dimensional elasto-plastic consolidation problem. 


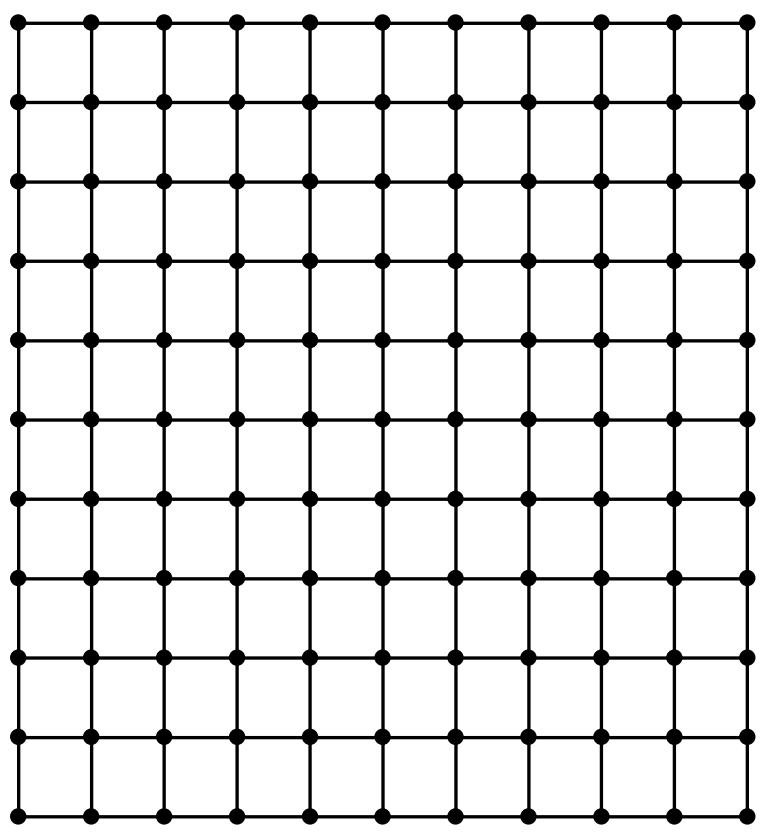

a. Grid 1 .

$\bullet \bullet \bullet \bullet \bullet \bullet \bullet \bullet \bullet \bullet \bullet \bullet \bullet \bullet \bullet \bullet \bullet \bullet \bullet \bullet \bullet \bullet \bullet \bullet \bullet \bullet \bullet \bullet \bullet \bullet \bullet \bullet \bullet \bullet \bullet \bullet$

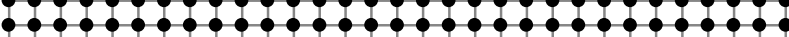
$0 \cdot 0 \cdot 0 \cdot 0 \cdot 0 \cdot 0 \cdot 0 \cdot 0 \cdot 0 \cdot 0 \cdot 0 \cdot 0$

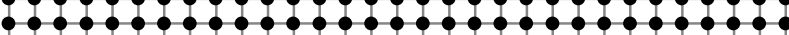

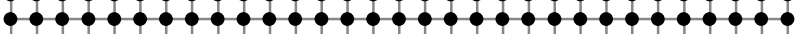

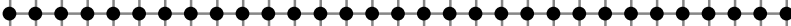

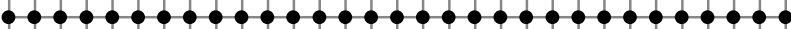
0 100 -

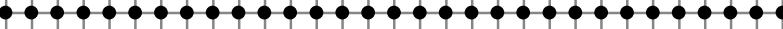
000000000000000000000000

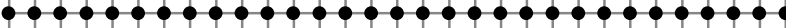

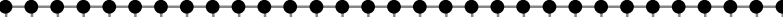

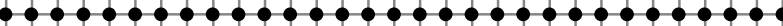

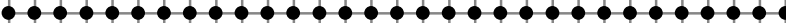
0000000000000000000

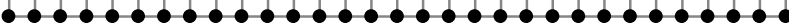
0 0

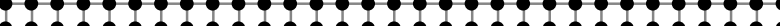
(1)

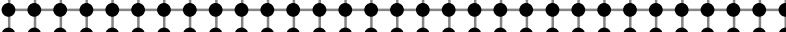
0. 00000000000000000000000.0 0000000000000000000

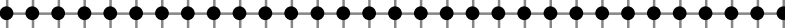

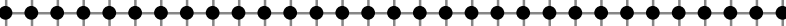

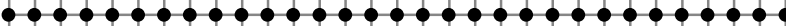

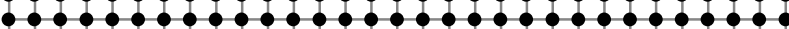

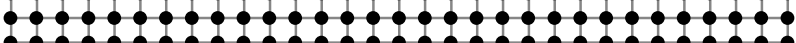

\section{c. Grid 3.}

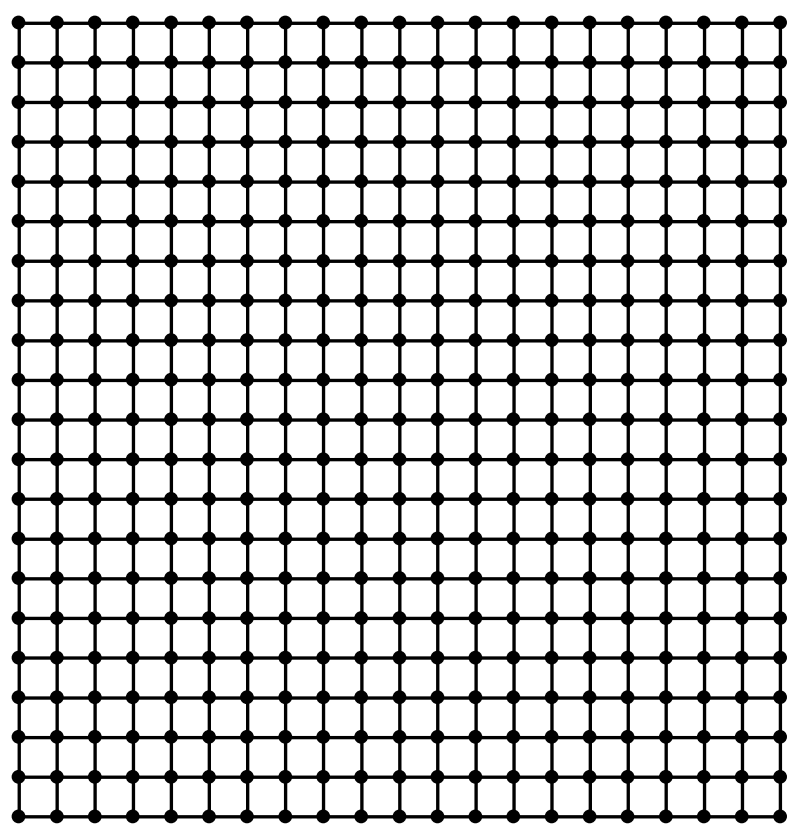

b. Grid 2.

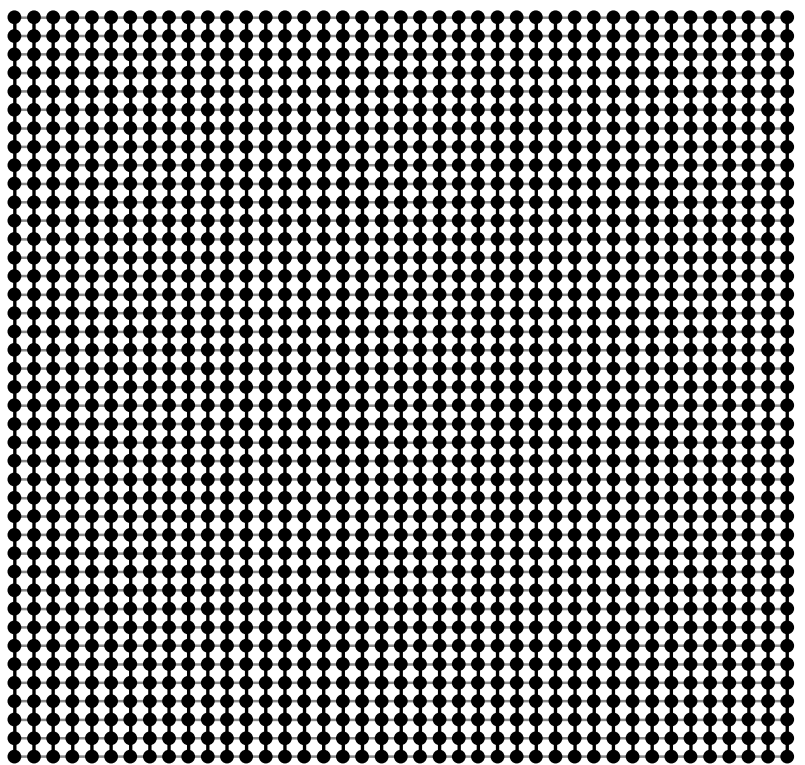

d. Grid 4.

Figure 7. MEM grids used for analysing two-dimensional elasto-plastic consolidation problem. 


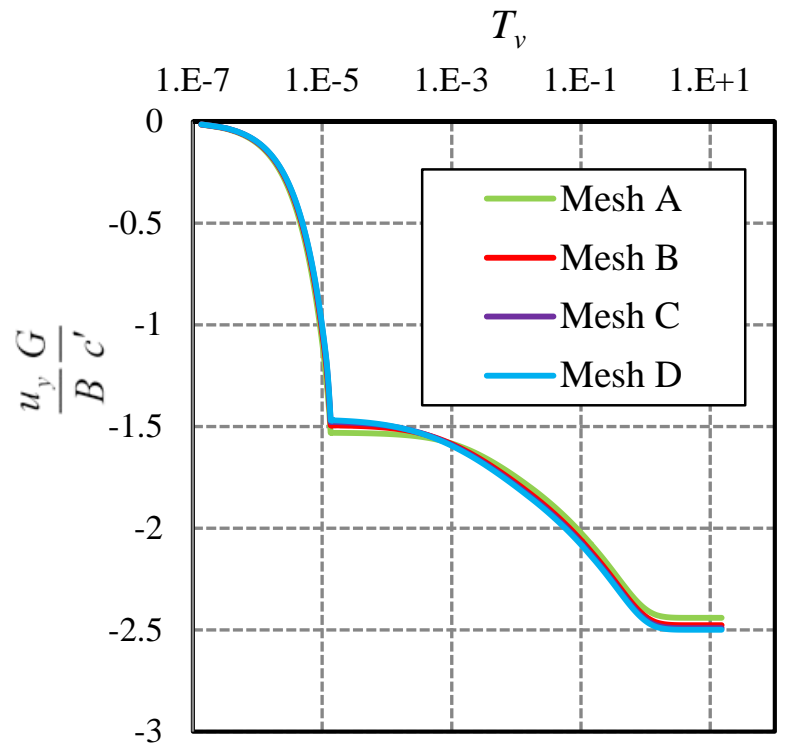

a. FE results, strip footing.

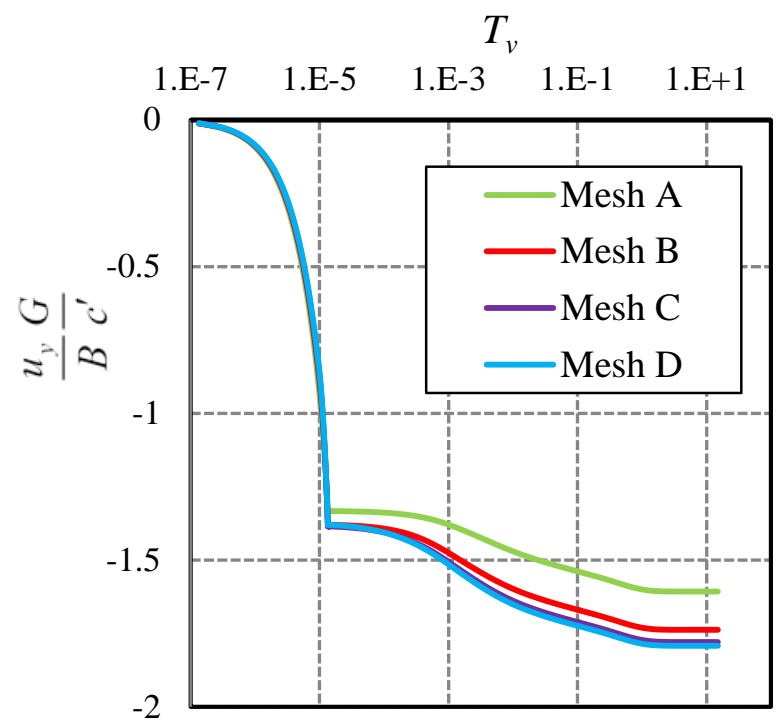

c. FE results, circular footing.

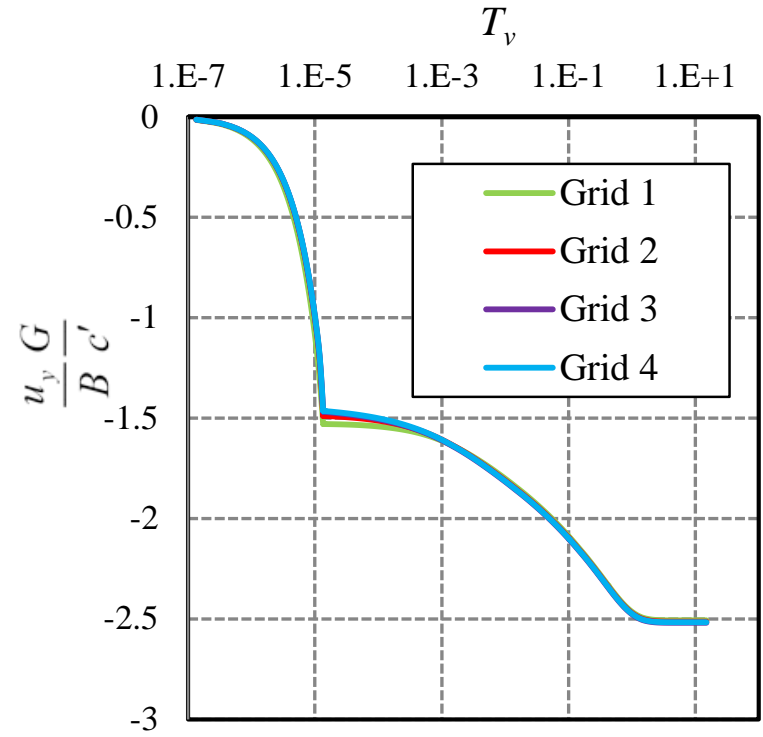

b. MEM results, strip footing.

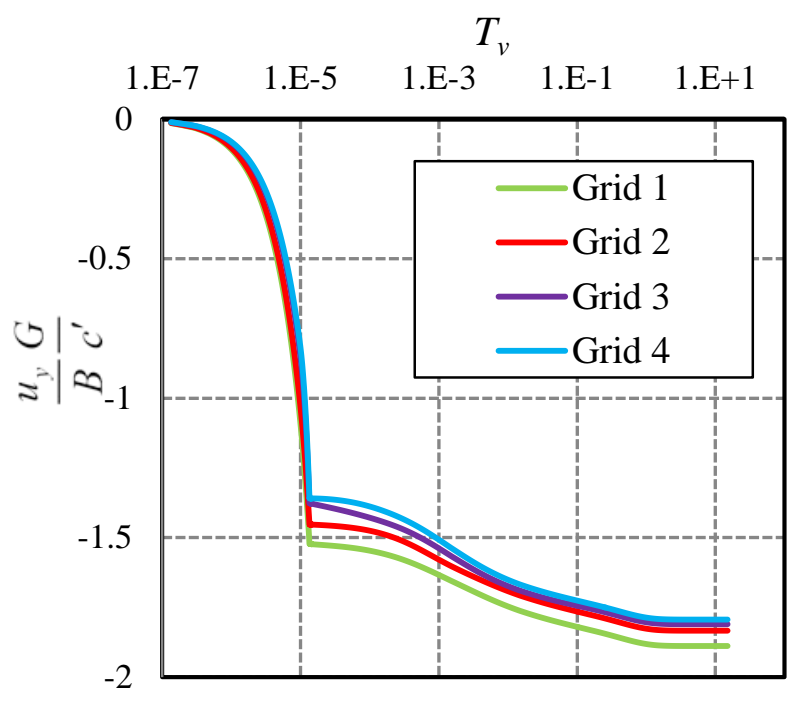

d. MEM results, circular footing.

Figure 8. Normalised settlement of footing centre versus normalised time. 


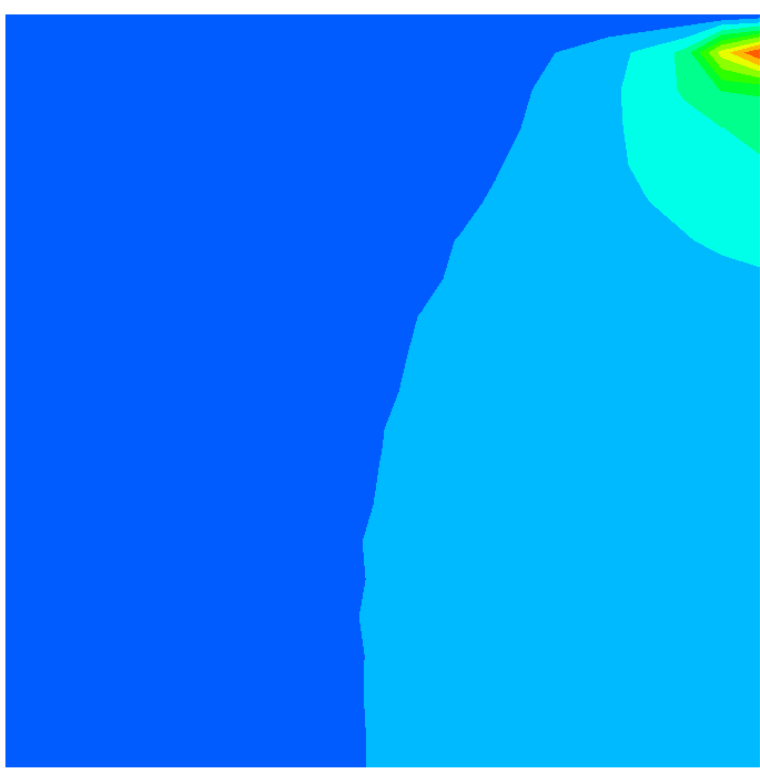

a. MEM Grid 2

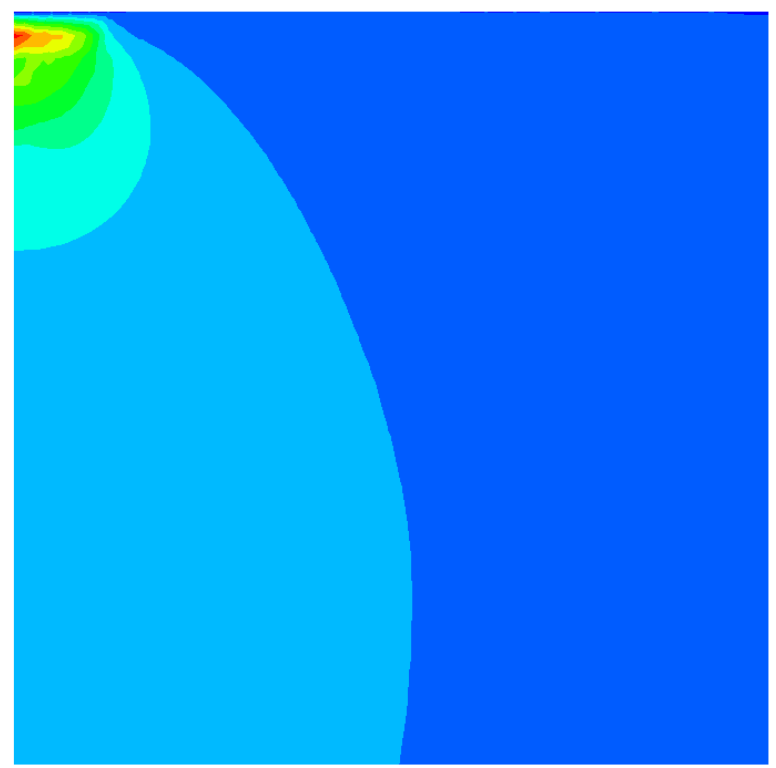

b. FE Mesh D

Figure 9. Contours of excess pore pressure normalised by the applied load at the end of loading phase (strip footing). 


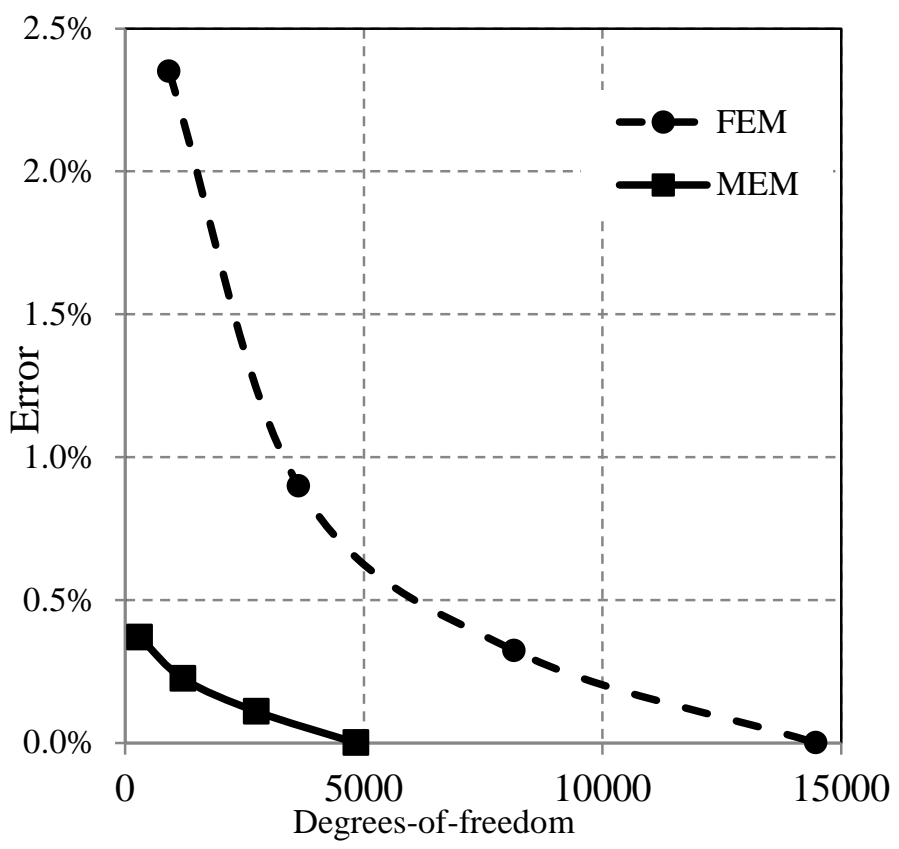

a. Error versus degrees-of-freedom, strip footing.

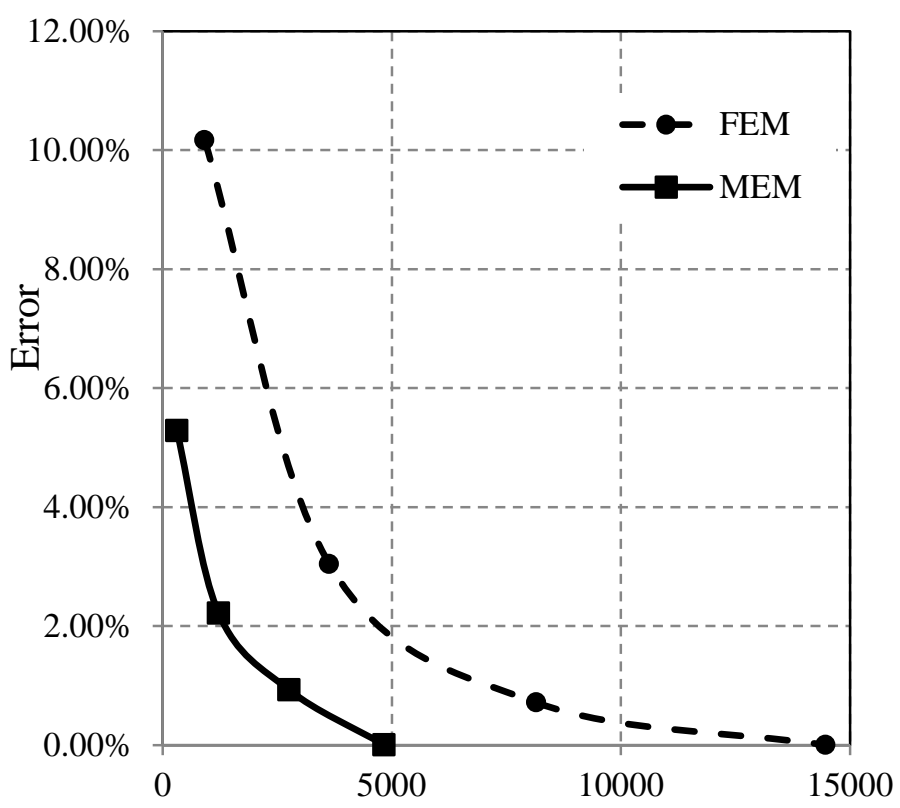

c. Error versus degrees-of-freedom, circular footing.

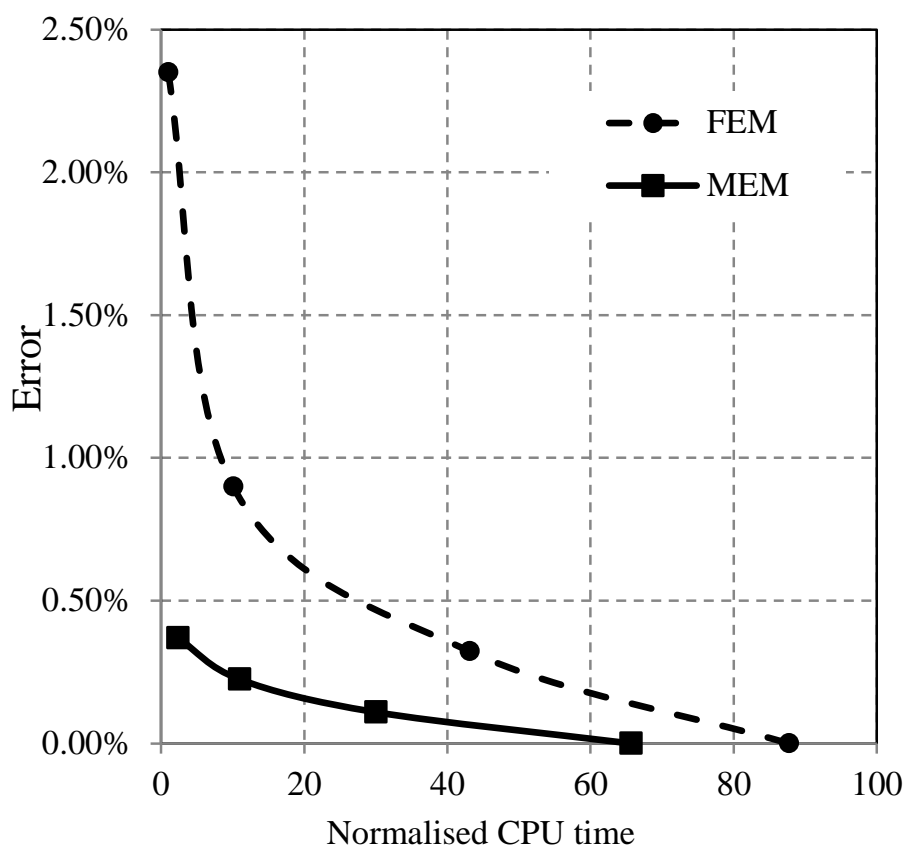

b. Error versus normalised CPU time, strip footing.

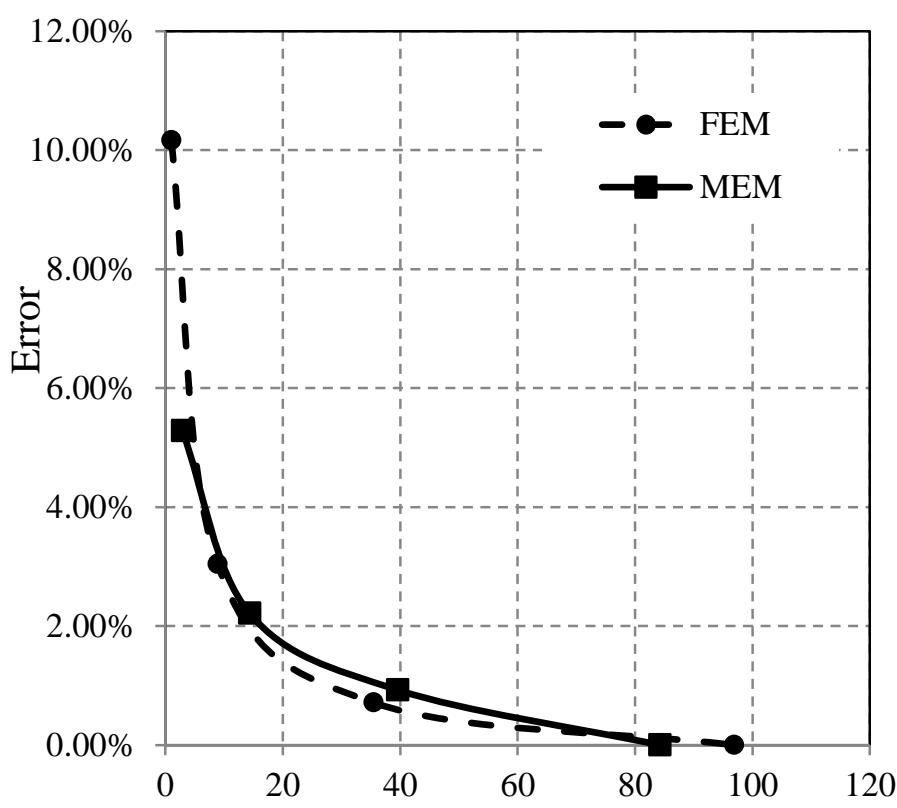

d. Error versus normalised CPU time, circular footing.

Figure 10. Performance of the MEM method versus the FE method. 


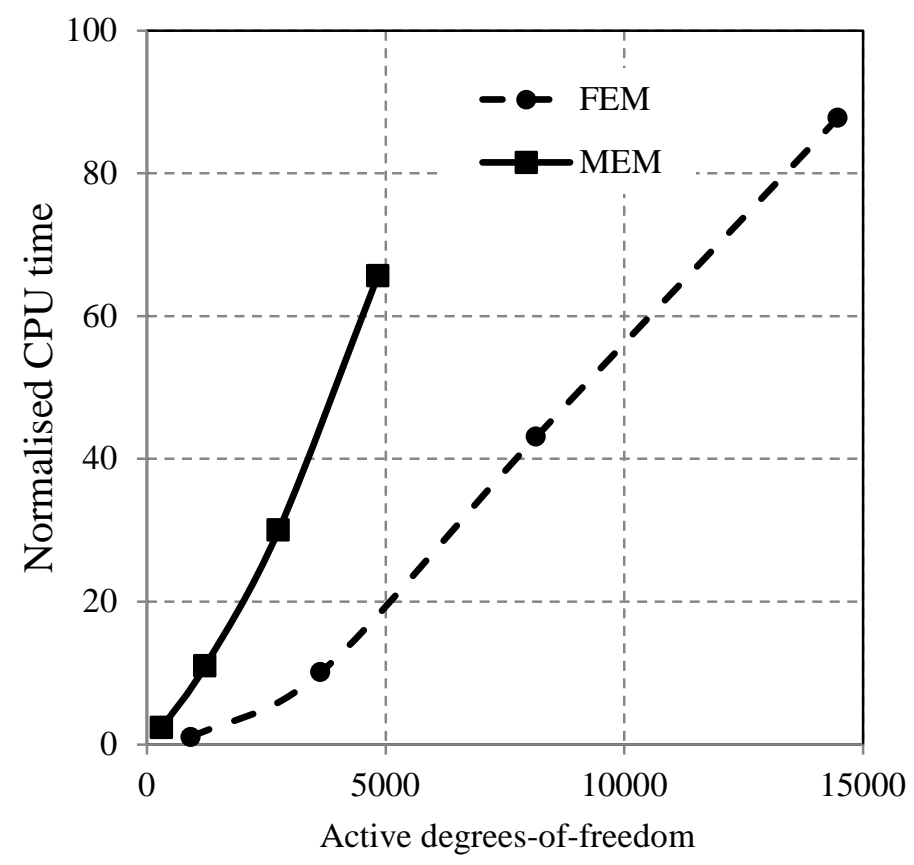

a. Strip footing.

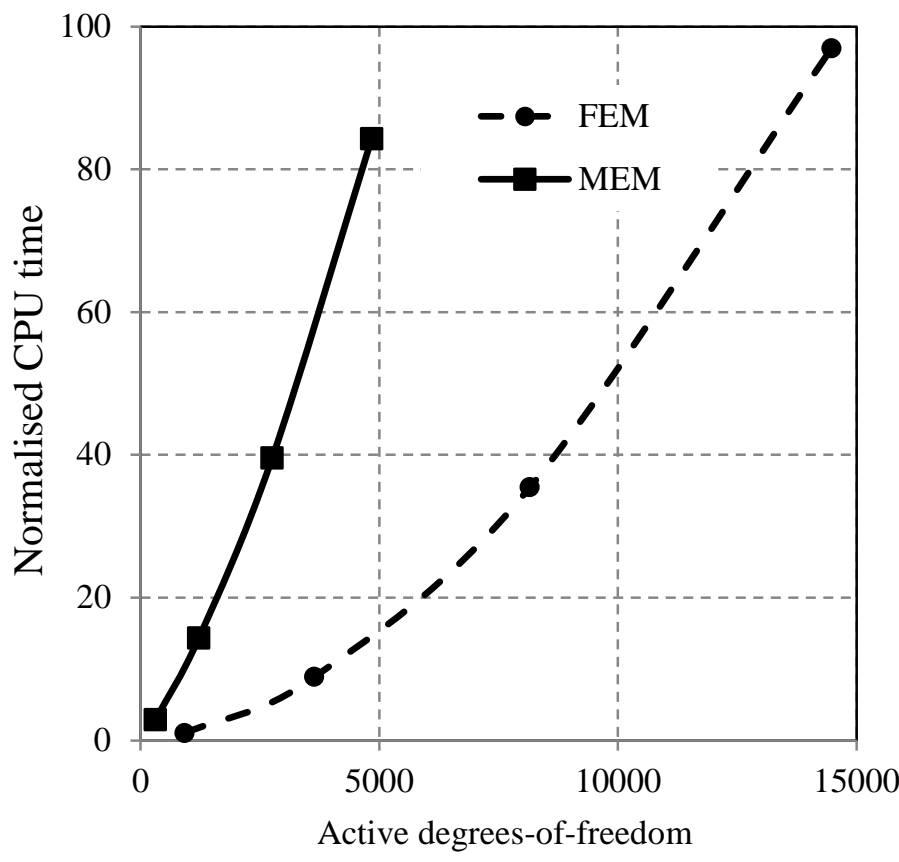

b. Circular footing.

Figure 11. Normalised CPU time versus degrees-of-freedom. 


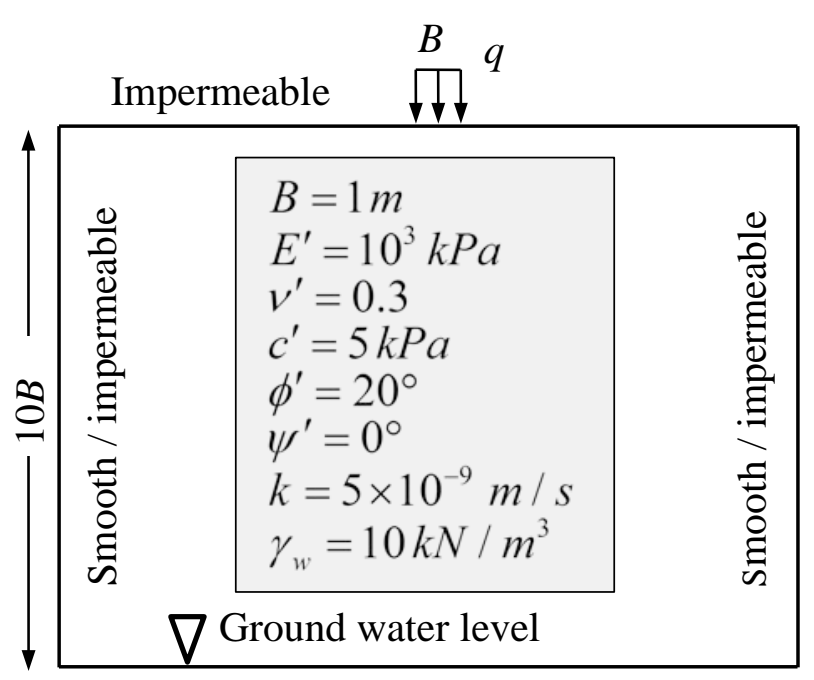

Smooth / impermeable

$20 B$

a. Problem domain, boundary conditions, and material properties.

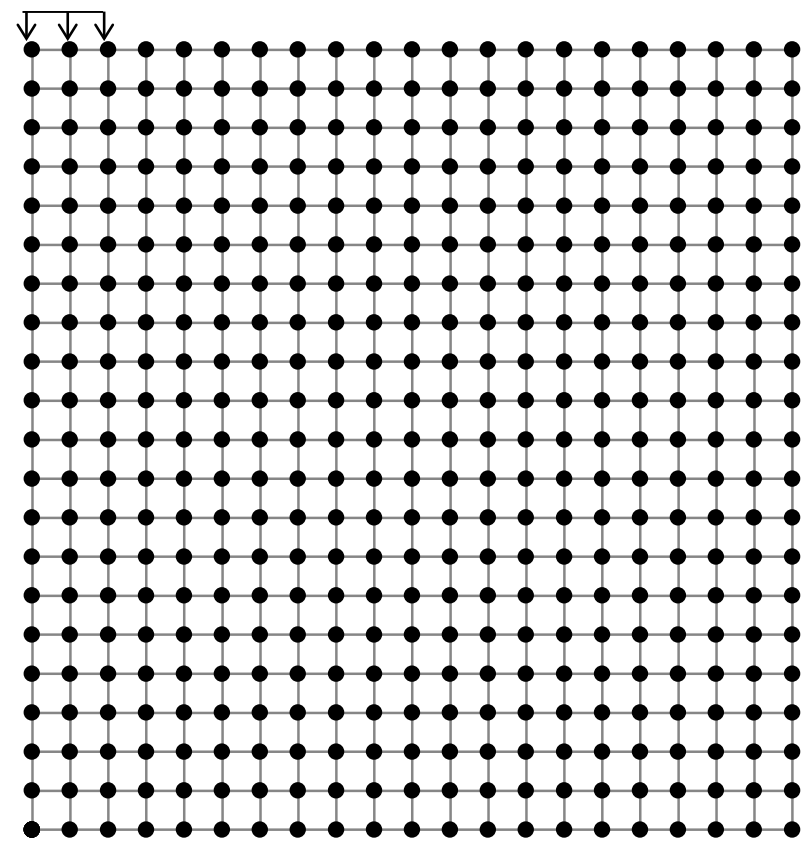

b. MEM uniform discretisation, 441 field nodes, 400 background cells.

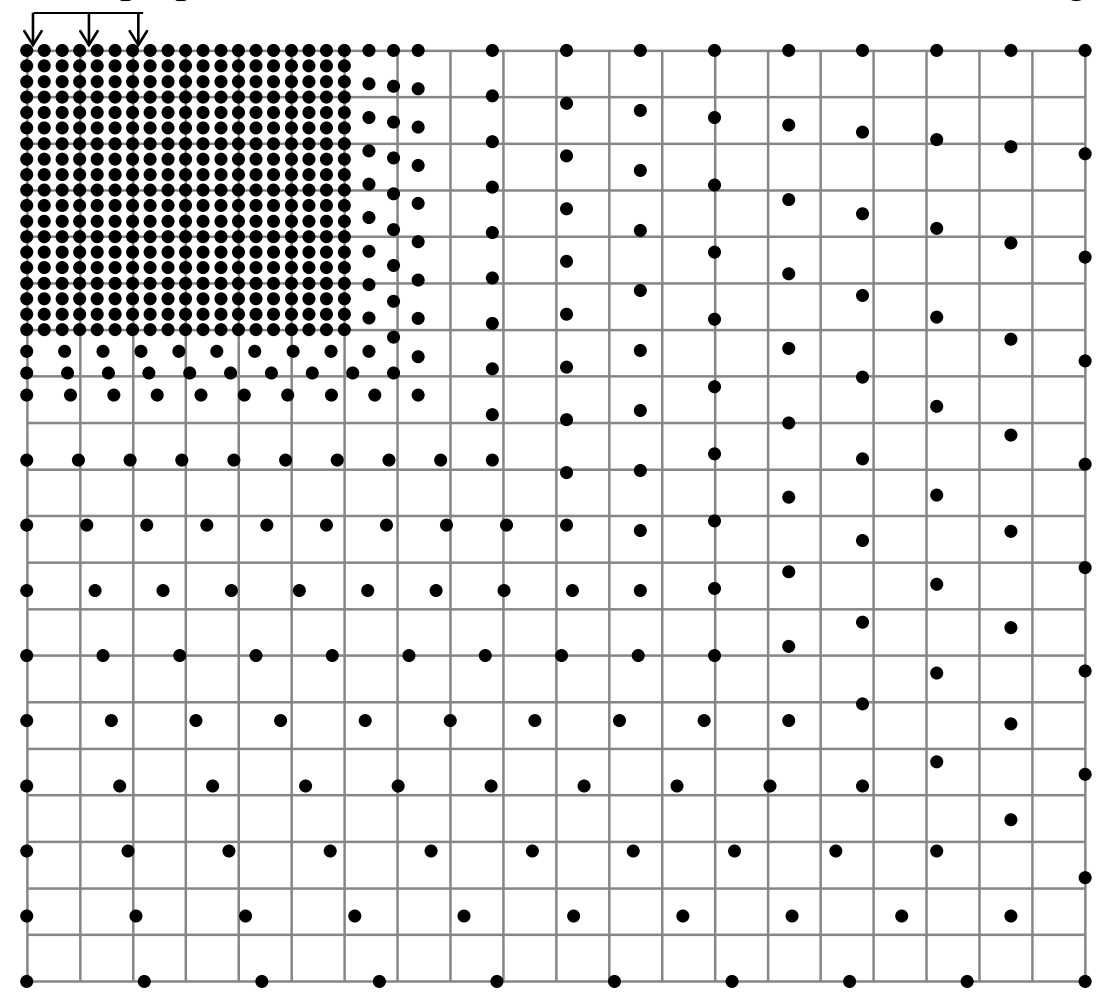

c. MEM non-uniform discretisation, 589 field nodes, 400 background cells.

Figure 12. Undrained bearing capacity of soil under a strip footing. 


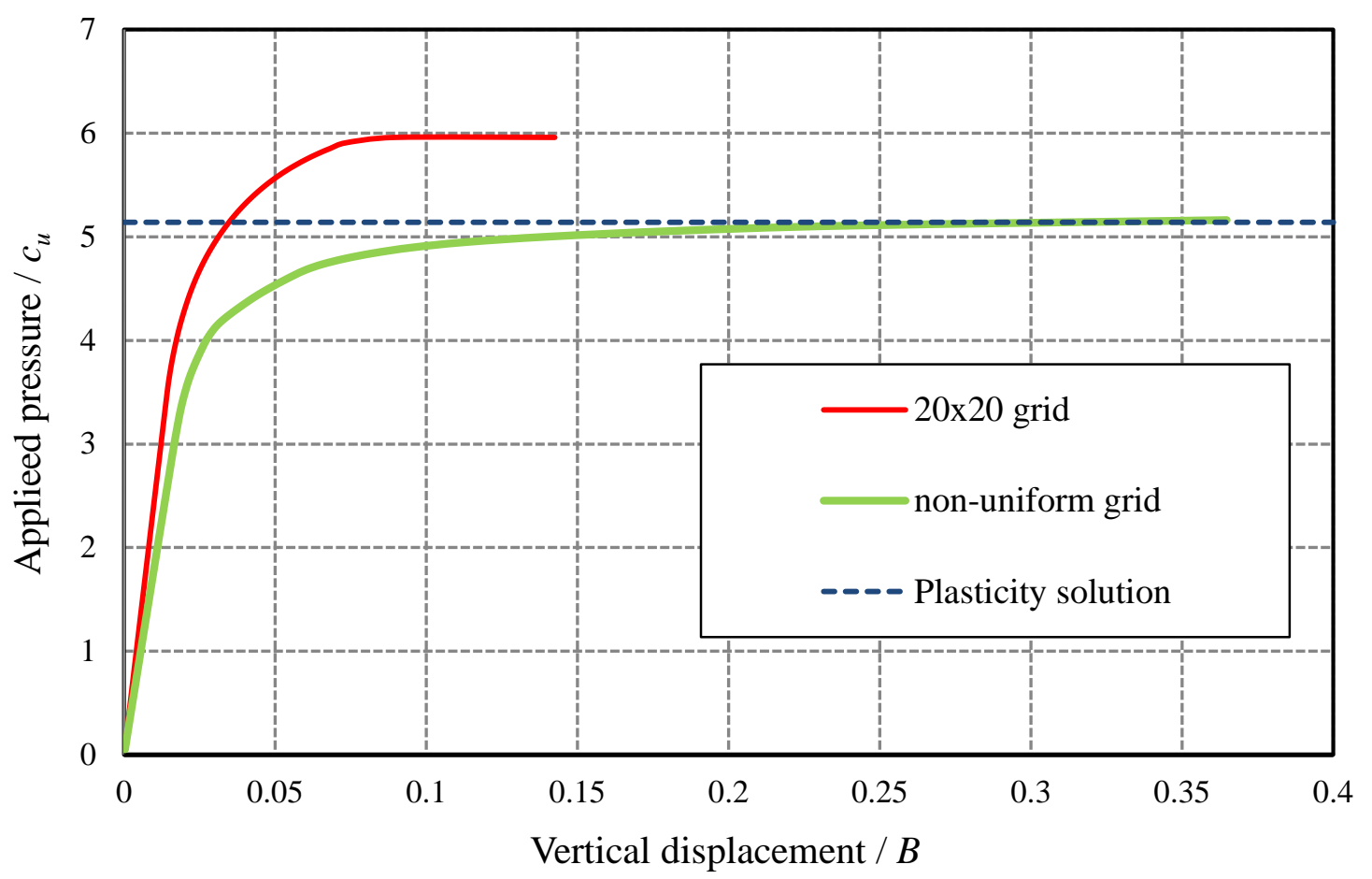

Figure 13. Normalised applied pressure versus normalised displacement. 


\begin{tabular}{|c|c|c|c|c|c|c|c|}
\hline Discretisation & $\begin{array}{l}\text { Elements (FE) } \\
\text { /Cells (MEM) }\end{array}$ & Nodal points & Gauss Points & $u_{i} / B$ (Strip) & $u_{f} / B$ (Strip) & $u_{i} /$ B (Circular) & $u_{f} / B$ (Circular) \\
\hline FE - Mesh A & 100 & 341 & 900 & 3.98 & 6.34 & 3.47 & 4.18 \\
\hline FE - Mesh B & 400 & 1281 & 3600 & 3.88 & 6.44 & 3.59 & 4.52 \\
\hline FE - Mesh C & 900 & 2821 & 8100 & 3.84 & 6.48 & 3.60 & 4.63 \\
\hline FE - Mesh D & 1600 & 4961 & 14400 & 3.82 & 6.50 & 3.59 & 4.66 \\
\hline MEM - Grid 1 & 100 & 121 & 900 & 3.98 & 6.52 & 3.96 & 4.91 \\
\hline MEM - Grid 2 & 400 & 441 & 3600 & 3.94 & 6.55 & 3.78 & 4.77 \\
\hline MEM - Grid 3 & 900 & 961 & 8100 & 3.82 & 6.54 & 3.63 & 4.70 \\
\hline MEM - Grid 4 & 1600 & 1681 & 14400 & 3.81 & 6.54 & 3.54 & 4.66 \\
\hline
\end{tabular}




\begin{tabular}{|c|c|c|c|c|c|}
\hline Discretisation & $\begin{array}{l}\text { Active degrees- } \\
\text { of-freedom }\end{array}$ & $\begin{array}{l}\text { Normalised CPU } \\
\text { time (strip footing) }\end{array}$ & $\begin{array}{l}\text { Error \% (strip } \\
\quad \text { footing) }\end{array}$ & $\begin{array}{l}\text { Normalised CPU time } \\
\text { (circular footing) }\end{array}$ & $\begin{array}{l}\text { Error \% (circular } \\
\text { footing) }\end{array}$ \\
\hline FE - Mesh A & 915 & 1.0 & 2.35 & 1.00 & 10.17 \\
\hline FE - Mesh B & 3636 & 10.09 & 0.90 & 8.89 & 3.04 \\
\hline FE - Mesh C & 8154 & 43.09 & 0.32 & 35.44 & 0.71 \\
\hline FE - Mesh D & 14472 & 87.73 & 0.00 & 96.89 & 0.00 \\
\hline MEM - Grid 1 & 309 & 2.36 & 0.37 & 2.89 & 5.28 \\
\hline MEM - Grid 2 & 1218 & 11.00 & 0.22 & 14.33 & 2.22 \\
\hline MEM - Grid 3 & 2756 & 30.00 & 0.11 & 39.56 & 0.93 \\
\hline MEM - Grid 4 & 4837 & 65.64 & 0.00 & 84.22 & 0.00 \\
\hline
\end{tabular}

